\title{
Helicopter Noise Reduction By Actively Controlled Flaps
}

\author{
Daniel Patt, Li Liu, and Peretz P. Friedmann ${ }^{\ddagger}$ \\ University of Michigan, Ann Arbor, MI 48109-2140 USA
}

\begin{abstract}
A detailed computational study was conducted to evaluate actively-controlled trailing-edge flaps as a noise reduction device. The helicopter noise problem is first introduced, noting that active control of blade-vortex interaction (BVI) noise is a relatively new field for computational research. Two structural models are considered for the present study; the first is a modal approach suitable for four-bladed hingeless rotors. The second is a finite element approach that is used to consider a five-bladed bearingless rotor. First, open-loop control is implemented with actively-controlled flaps (ACFs) and with conventional root-actuated individual blade control. The effectiveness of both techniques at noise reduction, vibration reduction, and rotor power reduction are considered in BVI descent flight. Subsequently, the BVI noise directivity in the near-field and far-field are examined for baseline and dual ACF noise control cases. Finally, reduction of noise and vibration is successfully demonstrated with a single plain flap configuration on a five-bladed bearingless rotor resembling that of the MD-900.
\end{abstract}

\section{Nomenclature}

$A_{n} \quad$ Control input amplitude for the $n^{\text {th }}$ harmonic

c Blade chord

$C_{P} \quad$ Rotor power coefficient

$C_{T} \quad$ Rotor thrust coefficient

$F_{H X n}, F_{H Y n}, F_{Z X n}$ Nondimensional $\mathrm{n} /$ rev hub shears

$M_{t r} \quad$ Trace Mach number, convenction speed of acoustic source along blade

$M H X n, M_{H Y n}, M_{Z X n}$ Nondimensional $\mathrm{n} /$ rev hub moments

$N_{b} \quad$ Number of rotor blades

$R \quad$ Rotor blade radius

$r \quad$ Spanwise position along the rotor blade $0 \leq r \leq R$

$V_{F} \quad$ Magnitude of free-stream velocity

Symbols

$\alpha \quad$ Rotor disk angle of attack

$\alpha^{\prime} \quad$ Effective rotor tip-path plane angle $\alpha$, corrected for wind tunnel effects

$\delta_{f} \quad$ Flap deflection angle

$\gamma \quad$ Lock number

$\gamma_{b} \quad$ Interaction angle between blade and vortex

$\mu \quad$ Helicopter advance ratio, nondimensional forward speed $\mu=V_{F} \cos \alpha / \Omega R$

$\Omega \quad$ Rotor angular speed

$\omega_{F 1} \quad$ First blade flapping frequency, nondimensionalized with $\Omega$

$\omega_{L 1} \quad$ First blade lead-lag frequency

$\omega_{T 1} \quad$ First blade torsional frequency

${ }^{*}$ Research Fellow, Member AIAA.

${ }^{\dagger} \mathrm{Ph} . \mathrm{D}$. Candidate, Student Member AIAA

$\ddagger$ Francois-Xavier Bagnoud Professor of Aerospace Engineering, Fellow AIAA 
$\begin{array}{ll}\phi_{c} & \text { Open-loop control phase angle } \\ \psi & \text { Rotor azimuth angle } \\ \sigma & \text { Rotor solidity } \\ \theta_{\mathrm{FP}} & \text { Flight path angle } \\ \theta_{t w} & \text { Distributed twist angle }\end{array}$

\section{Introduction and Objectives}

$\mathrm{R}$ OTORCRAFT noise is notoriously obtrusive, especially in low-speed descent flight conditions where heavy blade-vortex interaction (BVI) may be encountered. Community acceptance issues and military operational detectability concerns are driving research focused on the reduction of helicopter noise, under various flight conditions including the BVI regime. A number of active and passive methods for the control of rotorcraft noise have emerged, ${ }^{1}$ and various mitigation strategies are in operational use including passive low-noise design features and flight path modification. However, despite a number of studies suggesting that active strategies including actively controlled flaps (ACFs), ${ }^{2} \mathrm{HHC},{ }^{3}$ and conventional individual blade control (IBC) $)^{4}$ have the potential to reduce noise levels by $4-10 \mathrm{~dB}$ in certain flight conditions, these methods are only now in preliminary flight test stages. ${ }^{5}$ Concerns such as cost, interference with the primary flight controls, and the power usage of the system have prevented the commercial implementation of such devices.

The present study evaluates the actively controlled flap as a noise reduction device. The ACF has been proven to be effective at reducing vibrations ${ }^{6}$ in a number of flight conditions including high-speed forward flight $^{7}$ and low-speed descending flight in heavy BVI. ${ }^{8}$ Recently, the device has been shown to also be effective at reducing BVI noise, and it is even capable of simultaneous noise and vibration reduction. ${ }^{9}$ Flight tests of a helicopter equipped with a multiple ACF system is expected to further contribute to the acceptance of this emerging technology. ${ }^{5}$

The present paper is based upon a coupled aeroelastic/ aeroacoustic computational tool that has been extensively and successfully validated with data from the Higher-Harmonic Control Aeroacoustic Rotor Test (HART) program. ${ }^{3}$ The overall goal of the study is to evaluate different rotor and control configurations for their potential to reduce helicopter noise and further study the generation and propagation patterns of BVI noise, in both controlled and uncontrolled cases using the computational tool that has been developed.

The specific objectives of the paper are to:

1. Evaluate the effect of open-loop harmonic flap control inputs on noise, power, and vibration for two different hingeless rotor blade configurations similar to the MBB BO-105.

2. Evaluate the effect of open-loop conventional IBC inputs on noise, power, and vibration.

3. Evaluate noise propagation and directivity in the near and far-fields before and after closed-loop noise reduction for a $\mathrm{BO}-105$ rotor.

4. Achieve closed-loop reduction of noise and vibration for a five-bladed MD-900 rotor configuration with a single plain flap on each blade.

\section{Background}

TELICOPTER noise is generated by many different sources; however, this study is limited to BVI rotor 1 noise. The desire to reduce the annoyance of helicopter noise has motivated research aimed at understanding the mechanisms of propeller and rotor noise for more than sixty years. A general overview of rotorcraft noise and associated research through 1995 can be found in Ref. 10, where Schmitz discusses the different sources of noise from a helicopter and notes that the dominant source is the main rotor. The governing equations for noise radiation are introduced, and analytical considerations of radiation of different 
types of rotor noise are provided. This study also includes a variety of experimental helicopter noise data, studies of directionality and the influence of rotor flight condition on noise.

In the past ten years, progress has been made on several fronts in the research of helicopter noise. Analytical studies have continued to give insight into the generation and radiation characteristics of helicopter noise. ${ }^{11,12}$ Computational techniques have been refined, ${ }^{13}$ and new areas of computational research defined, including high-speed impulsive noise prediction, ${ }^{14,15}$ maneuvering flight noise prediction, ${ }^{16}$ and rotor broadband noise prediction. ${ }^{17,18}$ Finally, experimental and computational studies of active rotor noise reduction, particularly BVI noise, have begun to mature. ${ }^{1}$

Two analytical studies of BVI stand out as particularly insightful. Leishman uses wave tracing concepts to examine noise focusing from oblique and parallel blade-vortex interactions. ${ }^{11}$ Using a relatively simple analysis and physical arguments, Ref. 11 demonstrates BVI noise focusing and analyzes the effect of the BVI intersection angle on noise. Schmitz and $\mathrm{Sim}^{12}$ use simplified computational tools to study the geometric factors that govern BVI noise radiation. In this work, the authors classify BVI types on the advancing and retreating side, and study the associated acoustic radiation. The effect of advance ratio on noise was also considered over a limited range of $\mu=0.1$ and $\mu=0.3$.

In a recent review paper, Brentner and Farassat have given an overview of theoretical and computational noise prediction methods. ${ }^{13}$ The classical Ffowcs-Williams Hawkings formulation ${ }^{19}$ is discussed and several different forms are developed. Numerical techniques for rotor noise prediction are also reviewed including retarded-time, emission surface, and collapsing-sphere type algorithms. A limited discussion of recent developments for high-speed impulsive (HSI) noise and maneuvering rotorcraft noise prediction is provided. Another paper gives a more detailed description of a computational approach for maneuvering noise prediction. ${ }^{16}$ Recently, rotor broadband noise, caused largely by blade interactions with turbulence in the rotor wake, has been studied ${ }^{17}$ and compared with model data. ${ }^{18}$

In addition to this theoretical and computational research on noise generation, active noise reduction has emerged as a promising field, particularly in the BVI regime. The HHC and IBC approaches developed primarily for vibration reduction have also been studied as a means for reducing BVI noise. Testing of HHC for noise reduction started in the 1980's, and culminated in the HART ${ }^{3}$ and HART-II ${ }^{20}$ studies performed on a $40 \%$ scaled MBB BO-105 rotor. These produced extensive and high-quality data on the effect of openloop HHC inputs on BVI noise levels. These studies suggested that noise could be reduced by $4-6 \mathrm{~dB}$ on a carpet plane beneath the rotor with $0.85^{\circ}$ of $3 / \mathrm{rev}$ input phased at $38^{\circ}$. Interestingly, the control inputs most effective for noise reduction resulted in an increase in vibration levels and vice-versa, a relationship explored in Ref. 2. The HART studies also recorded vortex locations, and noted changes in wake geometry associated with the lower noise and vibration levels.

Individual blade control for noise reduction has also been explored, implemented with active pitch links and using active-twist rotor blades. Conventional root-actuated IBC was tested first on a full-scale MBв BO-105 rotor; ${ }^{21}$ later, a UH-60 rotor $^{4}$ was tested in the NASA Ames $40 \times 80$ wind tunnel. These studies indicated that reductions of 5-12dB were possible using open-loop control. Eurocopter Germany has performed closed-loop flight tests using IBC on a MBB BO-105. ${ }^{22}$ Booth and Wilbur recently conducted tests of an Active Twist Rotor (ATR) in the NASA Langley TDT wind-tunnel. ${ }^{23}$ Open-loop harmonic inputs were applied to the rotor, and reductions of $2-4 \mathrm{~dB}$ were obtained

Because of the difficulty of accurately predicting noise levels, most studies of active noise control have been experimental and the control was applied exclusively in the open-loop. Furthermore, many computational studies associated with noise simulation focused only on correlation with experimental data, especially HART data. Malovrh and Gandhi ${ }^{24,25}$ performed a study on the effect of IBC on individual bladevortex interactions on the advancing and retreating sides. This study used a comprehensive rotor code, similar to UMARC, developed by Tauszig. ${ }^{26}$ A variety of localized, non-harmonic open-loop control inputs with magnitudes as high as $3^{\circ}$ were selected, and resulted in noise reductions of up to $8 \mathrm{~dB}$. An attempt was made to consider the acoustic contribution of individual BVI events, ${ }^{25}$ however, closed-loop noise control was not considered.

More recently, the authors have presented an extensive study using active closed-control and single and dual ACFs in a series of publications. ${ }^{2,27,28}$ Noise reductions of $4-5 \mathrm{~dB}$ could be achieved. Simultaneous 
noise and vibration reduction was also considered. ${ }^{2}$

The studies mentioned above indicate that the understanding of the BVI noise problem has increased considerably during the past ten years. Notably, by considering the work found in Refs. 2,3,11,12 and 25, the primary factors governing noise emission from a BVI event can be identified:

1) The advance ratio, rate of descent, and rotor angular speed all affect the geometry of the trailed wake, and thus the strength and type of BVI. Noise from BVI is most severe when the wake is trailed directly into the plane of the rotor and oncoming blades.

2) The magnitude of pressure fluctuations on the rotor blade have a strong effect on the magnitude of BVI noise and vibration produced. Subsequently, circulation strength and trajectory of the vortex segment may be influenced.

3) The miss distance between a vortex segment and the oncoming rotor blade can enhance the severity of a BVI event as the miss distance becomes smaller.

4) The interaction angle $\gamma_{b}$ between the vortex segment and blade in the plane of the rotor (whether an interaction is parallel or not) can alter the magnitude of BVI noise, the directionality of the noise, and the propagation efficiency.

Active control has the potential to mitigate noise by altering any of the latter three parameters, but recent studies have identified the interaction angle as the dominant parameter governing BVI noise reduction. ${ }^{1,2,25}$ When an interaction is nearly parallel, acoustic propagation characteristics may be changed drastically from those of perpendicular interactions, resulting in a significant increase of the annoyance to observers on the ground.

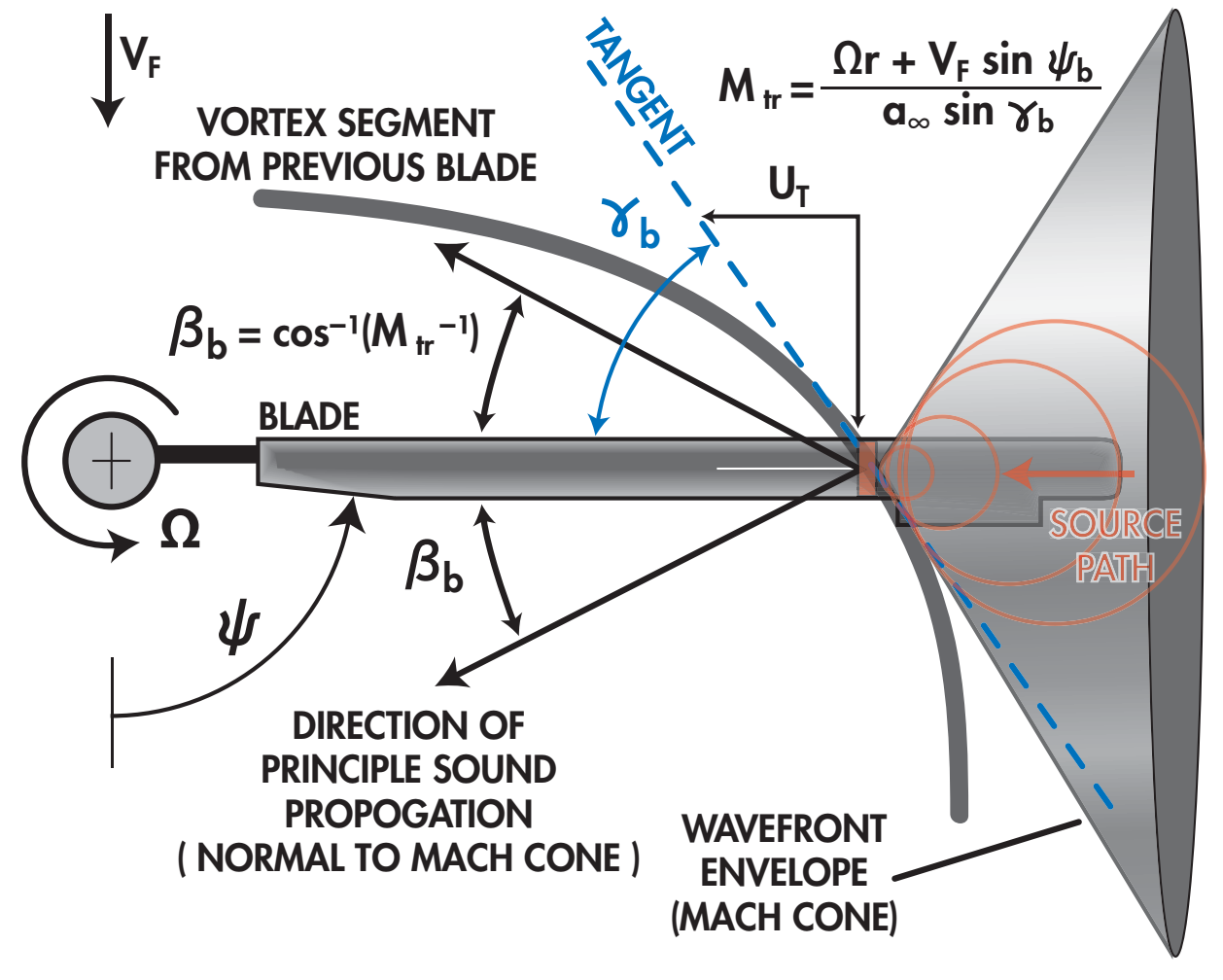

Figure 1. Interaction angle of a blade-vortex encounter.

The effect of the interaction angle on noise propagation is most effectively demonstrated in Refs. 11 and 12. Following the procedure of Ref. 11, consider the geometry of a BVI event as depicted in Fig. 1. The trace 
Mach number (nondimensional convection speed) is introduced, defined as follows:

$$
M_{t r}=\frac{\Omega r+V_{F} \sin \psi}{a_{\infty} \sin \gamma_{b}}
$$

In physical terms, the trace Mach number can be regarded as the speed of a BVI event, representing the movement of the aerodynamic force distribution (as acoustic sources) at interactions. Strong phase accumulation (lumped acoustic disturbance) occurs when trace Mach numbers become supersonic over a significant portion of the blade. Interactions with a supersonic trace mach number are acoustically efficient in propagating in certain directions. In fact, some research has speculated ${ }^{29}$ that some parallel acoustic events have more 2-D like decay characteristics, falling off as an inverse first power rather than an inverse second power. Experimental evidence of this departure from inverse-square decay was first noted in Ref. 30, a wind-tunnel test of a scaled MBB BO-105 rotor. Additional discussion of this trend, including simple analytical models suggesting that this slower decay only occurs in the far-field is presented in Ref. 31 . The denominator of Eq. (1) implies that a near parallel interaction $\left(\gamma_{b} \approx 0\right)$ will have a high trace $\left(M_{t r} \geq 1\right)$ mach number. It is interesting to note that a computational study evaluating the contributions of individual BVI events ${ }^{25}$ has suggested that among the several interactions on the advancing side, only the parallel or nearly parallel interactions are major contributors to noise in the far-field, which tends to support the observation made for Eq. (1). This finding was also supported by the results of Ref. 2.

Although the understanding of the BVI acoustic problem has increased considerably, much research on the implementation of active control devices on helicopters is still required. In particular, different control techniques and rotor configurations need to be compared and evaluated, and methods for durable and economical implentation must be developed.

\section{Description of Simulation}

\section{A. Structural Dynamic Models}

TWO rotor configurations are considered in the present study, as illustrated in Fig. 2 The blade properties 1 for these configurations are given in Table 1. The first configuration is described extensively in Refs. 2 and 6. The four-bladed hingeless rotor shown in Fig. 2 a resembles a MBB BO-105 rotor. Each blade has a root offset $e$, and it rotates with constant angular speed $\Omega$. Each blade has fully-coupled flap, lead-lag, and torsional dynamics, and includes nonlinearities due to moderate blade deflections.

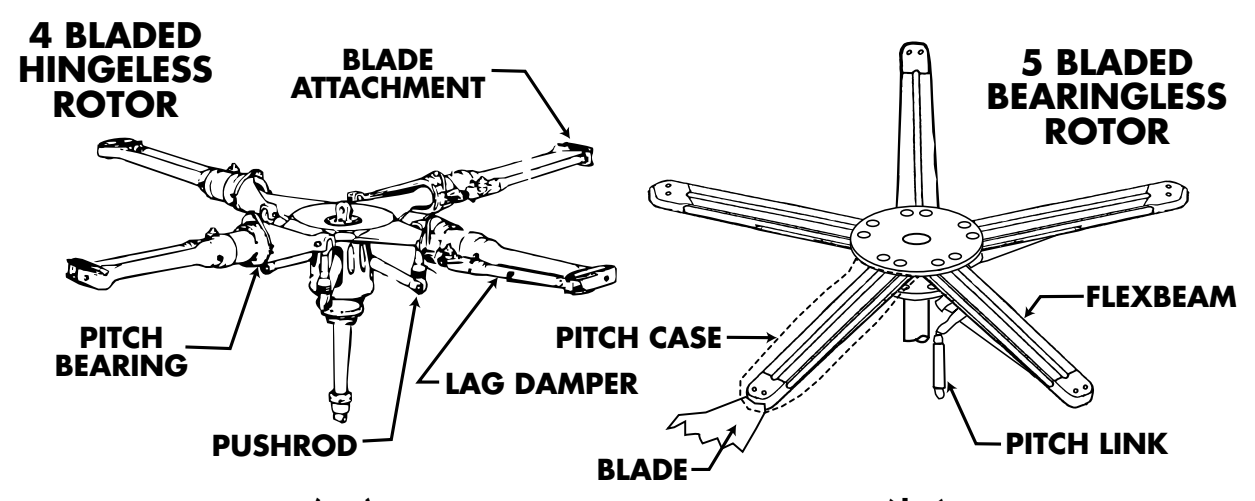

(a.)

(b.)

Figure 2. Hingeless and bearingless rotor hubs

The second configuration is a five-bladed bearingless rotor that resembles the MD-900 Explorer rotor, as used in the Boeing smart material actuated rotor technology (SMART) tests. ${ }^{32}$ The structural dynamic model is based on a composite blade model developed by Yuan and Friedmann ${ }^{33}$ which is capable of modeling transverse shear deformations, cross sectional warping and swept tips. The equations of motion are 
formulated based on the finite element discretization of Hamilton's principle and assuming moderate deflections. The blade is discretized by beam type elements, having 23 nodal degrees of freedom. Originally this model was applied to hingeless blades. Therefore, in the present study the structural dynamic model was modified to properly represent the multiple load paths by including the pitchcase elements that are an important part of the bearingless rotor design. The resulting finite element model obtained after this modification is quite similar to a bearingless rotor configuration generated in RCAS (the Rotorcraft Comprehensive Analysis System), a rotorcraft simulation tool, also based on the finite element method. ${ }^{34}$ Since the present structural dynamic model and the RCAS model are quite similar, the bearingless rotor blade model shown in Fig. 3 captures the essence of both models. The RCAS model was used to correlate natural frequencies with the results obtained by the current analysis, as presented next. The snubber spring and damper elements in the RCAS model are replaced by concentrated stiffness and damping at the blade root, while the control slide is not represented in this analysis.

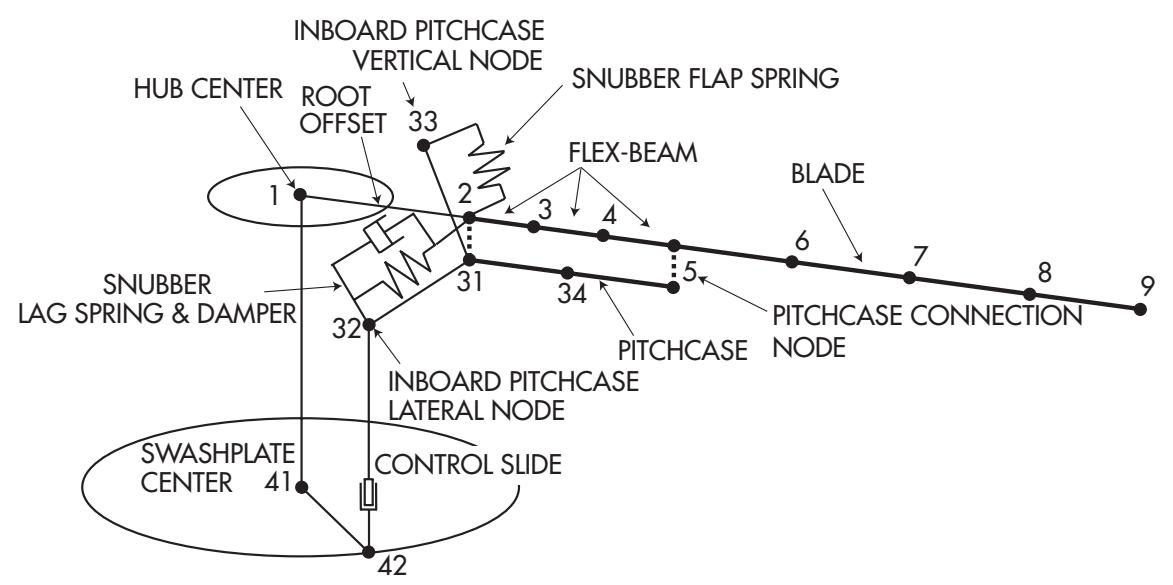

Figure 3. RCAS finite element model for a bearingless rotor

The flexbeam portion of the rotor hub is modeled by beam elements and cantilevered to the hub at Node 2, as illustrated in Fig. 3 . The pitchcase is also modeled by beam-type elements, forming a second load path. The inboard end node (Node 31) of the pitchcase is restrained to the hub through the snubber-damper assembly, modeled by linear lead-lag and flap springs, and a lead-lag damper, with spring constants and damping coefficients determined experimentally. This node is also connected to the pitch link, where the pilot pitch control is applied. At the blade-flexbeam-pitchcase junction (Node 5), continuity is imposed for both displacements and slopes for flap and lead-lag bending and displacements of other degrees of freedom.

Note that the bearingless blade model includes multiple load paths from beam-type elements for the pitchcase and enforcing continuity at the blade/pitchcase connection. In the actual MD-900 model, 12 spatial elements are used to model the flexbeam/blade combination, and 2 elements model the pitchcase. Modal reduction with eight coupled natural rotating modes (four flap, two lead-lag, one torsion and one axial) is used to solve the aeroelastic equations of motion.

\section{B. Aerodynamic and Acoustic Models}

THE aerodynamic and acoustic solution models used for the simulation are described in Refs. 2, 27, and 128 . A new method for accurately and efficiently computing unsteady pressure distribution over a blade was developed in Ref. 27. An enhanced wake model was developed and described in Ref. 27. This was combined with the development of a noise prediction capability based on WOPWOP ${ }^{35}$ which accounts for fully flexible blades with the blade dynamics that are consistent with the structural model. ${ }^{27}$ 


\begin{tabular}{llll}
\hline Parameter & & CONFIG-I & CONFIG-II \\
Type & & MBB BO-105 & MD-900 \\
Flaps & & $1-2$ Servo & 1 Plain \\
$N_{b}$ & 4 & 5 \\
$\Omega(\mathrm{RPM})$ & 425 & 392 \\
$R(\mathrm{~m})$ & 4.91 & 5.16 \\
$c / R$ & 0.05498 & 0.04924 \\
$\omega_{F 1}$ & 1.123 & 1.04 \\
$\omega_{L 1}$ & 0.732 & 0.64 \\
$\omega_{T 1}$ & 3.17 & 5.67 \\
$\theta_{t w}$ & $-8^{\circ}$ & $-10^{\circ}$ \\
$\theta_{\mathrm{FP}}$ & $6.5^{\circ}$ & - \\
$\alpha^{\prime}$ & - & $-3.5^{\circ}$ \\
$C_{W}$ & 0.005 & 0.006 \\
$\sigma$ & 0.07 & 0.078 \\
$\gamma$ & 5.5 & 5.6 \\
precone angle & $2.5^{\circ}$ & $3^{\circ}$ \\
\hline
\end{tabular}

Table 1. Rotor configurations

\section{Validation of Simulation}

N extensive structural, aerodynamic, and acoustic validation study was previously conducted using

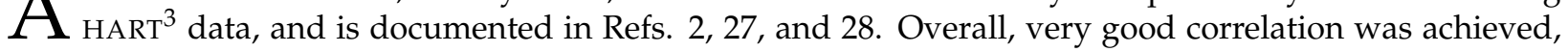
featuring remarkable reproduction of acoustic signatures at locations around the rotor for the baseline case. This validation effort was for a 40\% scaled MBB BO-105 hingeless rotor. In this section, the bearingless rotor structural model, resembling the MD-900 SMART rotor, is correlated with results generated by two comprehensive rotor codes.

The natural frequencies and mode shapes of the linear, undamped system in a vacuum are first calculated and compared to those obtained using RCAS, as shown in Table 2 The comparisons are quite good.

\begin{tabular}{c|cc} 
& Current Simulation & RCAS \\
\hline$\omega_{L 1}$ & 0.654 & 0.654 \\
$\omega_{F 1}$ & 1.043 & 1.048 \\
$\omega_{F 2}$ & 2.573 & 2.572 \\
$\omega_{L 2}$ & 3.488 & 3.498 \\
$\omega_{F 3}$ & 4.472 & 4.473 \\
$\omega_{T 1}$ & 5.667 & 5.409 \\
$\omega_{F 4}$ & 7.270 & 7.273 \\
$\omega_{A 1}$ & 25.70 & 25.82
\end{tabular}

Table 2. Natural frequency comparison, MD-900 type rotor

In order to validate both the blade response and flap effectiveness of the bearingless rotor, predictions of tip pitch deflection and blade torsional moment are calculated. The results generated by the simulation for 
a baseline (no control) case and an active control case with prescribed flap deflections are compared against the results obtained using the comprehensive rotorcraft code CAMRAD II. ${ }^{36,37}$ The baseline case simulates descent flight condition at $\mu=0.2$ with a thrust coefficient of $C_{T} / \sigma=0.0774$. The rotor is trimmed to zero first-harmonic blade flapping and the specified $C_{T} / \sigma$ using a wind-tunnel type trim procedure. ${ }^{2,28}$ For the controlled case, the flap deflection is prescribed at a single frequency of $4 / \mathrm{rev}$ with a maximum $2^{\circ}$ deflection amplitude, $\delta_{f}=2 \cos (4 \psi-240)$.

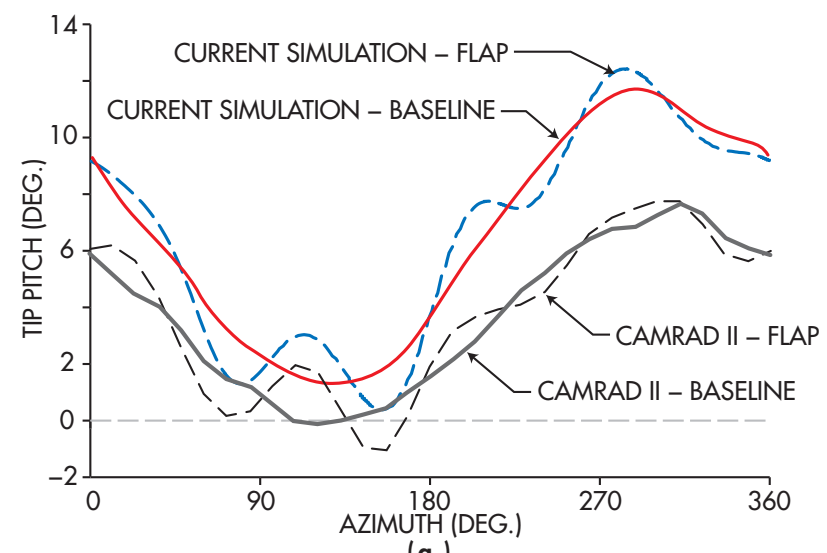

(a.)

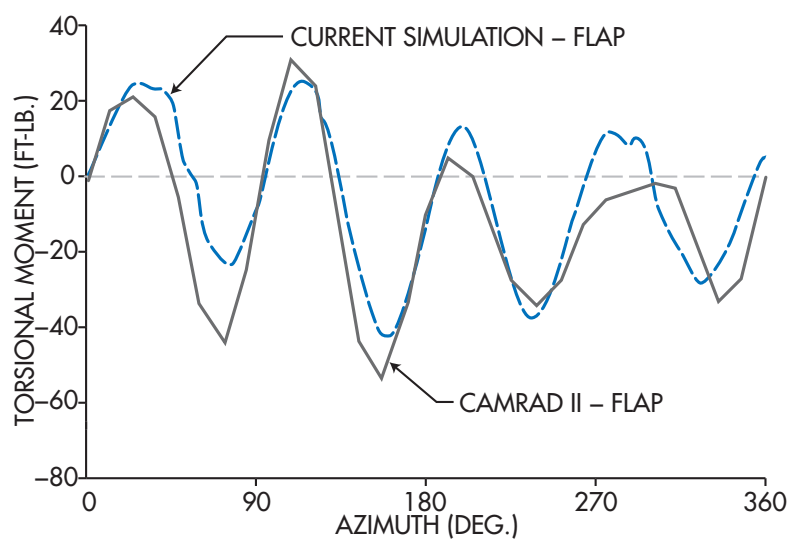

(b.)

Figure 4. Simulation correlation with CAMRAD II

The blade tip pitch response (torsion, collective, and cyclic, but excluding built-in pretwist) for the baseline and controlled cases are compared in Fig. 4a. The general trends of pitch variation with azimuth for both the baseline and controlled cases are very similar, except that the current simulation exhibits a nearly constant offset angle from the CAMRAD II predictions. This difference may be attributed to the fact that the cambered airfoils used on the actual MD-900 rotor and in the CAMRAD II predictions are replaced by an uncambered airfoil for the present simulation. Note that the $2^{\circ}$ of prescribed flap deflection induces roughly the same magnitude of total blade tip pitch deflection in the CAMRAD II prediction and the current simulation.

Blade torsional moments at a $40 \%$ radial location are compared in Figure 40 , with the same prescribed flap deflection. The predictions of current simulation agree reasonably well with those of CAMRAD II.

\section{Results}

$\mathrm{T}$ HE effectiveness of the active flaps for noise reduction, as well as modification of rotor vibration and power are examined in this section. An open-loop control study is performed for two four-bladed hingeless flapped rotor configurations with different blade torsional stiffnesses. The effect of the flap on noise, power and vibration is considered. A similar open-loop study is also performed for the same rotor equipped with a conventional root actuated IBC system. Subsequently, the directionality of noise around the rotor is examined for the baseline condition as well as active noise reduction case utilizing the dual-flap ACF system. Finally, noise reduction, vibration reduction, and simultaneous reduction are considered for a five-bladed bearingless rotor configuration with plain flaps, which differs from the servo flap configuration used in all other cases.

\section{A. Open-loop noise, power, and vibration response for BO-105 rotor with a flap}

DREVIOUS work has demonstrated the capability of the ACF for both noise and vibration reduction using 1 an adaptive HHC controller. ${ }^{2}$ The effect of active flap deflection on rotor power as well as noise and vibration is addressed in this section. Cheng and $\mathrm{Celi}^{38}$ performed a study of $2 / \mathrm{rev}$ HHC inputs using a 
simple rotor aeromechanics model. The study noted that rotor power reductions were possible when using properly phased open-loop HHC input at a level flight condition, and at a relatively high advance ratio of $\mu=0.3$. Reference 38 was based on table look-up aerodynamics and a nonlinear drag model; and it was emphasized that power reductions could only be simulated when using the nonlinear drag model. A subsequent study by the same authors included a dynamic inflow model. ${ }^{39}$ With the addition of dynamic inflow, the amount of power reduction that could be simulated was reduced significantly.

Reduction in rotor power using ACFs has not been considered to date. Therefore, the power reduction that can be achieved with an ACF system operating in the open-loop mode is studied in this section. The results presented are for a single flap configuration operating in descending flight with $\mu=0.15$. Two blade configurations are considered: 1) the MBB BO-105 type blade as described in Table 1. and 2) a blade that is very soft in torsion with $\omega_{T 1}=2.50$ and $\omega_{T 2}=6.98$, but having all other properties identical to blade 1 . The open loop inputs are at frequencies of 2,3,4 and 5/rev with varying phase. The present study incorporates a free-wake model as well as a drag model with a linear correction for flap deflection. ${ }^{27,28}$

An open-loop phase sweep was conducted to determine the effect of flap deflections on the rotor power, BVI noise, and the vertical $4 / \mathrm{rev}$ vibratory hub shear $F_{H Z 4}$. Using a constant half peak-to-peak flap deflection amplitude of $A_{n}=4^{\circ}$, control phases $\left(\phi_{c}\right)$ were tested in $30^{\circ}$ increments for each of the 2,3,4 and 5/rev harmonics at the default $\mu=0.15$ descent flight condition, as shown in Eq. (2):

$$
\delta_{f}(\psi)=A_{n} \cos \left(n \psi-\phi_{c}\right)=A_{n}\left[\sin \left(\phi_{c}\right) \sin (n \psi)+\cos \left(\phi_{c}\right) \cos (n \psi)\right]
$$

It is expected that the increase in drag due to flap deflection will cause increases in the power required. Average rotor power is defined as the instantaneous power required to drive the rotor at a constant angular velocity $\Omega$ averaged over one revolution,

$$
C_{P}=\frac{\Omega}{2 \pi} \int_{0}^{2 \pi}-C_{M_{H z 1}}(\psi) d \psi,
$$

where $M_{H z 1}$ is the total yawing moment about the hub and includes the effect of unsteadiness, compressibility, dynamic stall (if applicable), and the additional drag due to flap deflection. The negative sign in front of $C_{M_{H z 1}}(\psi)$ is due to the fact that it represents the torque about the rotor shaft due to the loading on the blades, and therefore the engine must supply a torque equal to $-C_{M_{H z}}(\psi)$ to maintain a constant angular velocity. ${ }^{6}$ Equation 2 is a general expression valid for blades with or without actively controlled flaps.

Changes in right-rear skid microphone noise levels corresponding to the different control harmonics and phases are shown in Fig. 5. At this flight condition, every harmonic is capable of noise reduction if properly phased. Reductions of almost $7 \mathrm{~dB}$ are possible with $3 /$ rev input and $5 \mathrm{~dB}$ with $2 /$ rev input.

Figure 6 shows the resulting changes in required rotor power for the MBB BO-105 type blade with $\omega_{T 1}=3.17$. Notably, there is no control input capable of reducing the rotor power required. At near $0^{\circ}$ of phase with $3 / \mathrm{rev}$ inputs, a significant power penalty is encountered. On the other hand, near $180^{\circ}$ for $3 /$ rev, there is almost no power penalty.

The effects of flap deflection on vibration levels are shown in Fig. 7 It is apparent from this figure that any of the four tested control harmonics is capable of reducing vibrations from the baseline if the control input is properly phased. A reduction of $90 \%$ can be achieved with $3 / \mathrm{rev}$ alone.

An identical open-loop study was performed for a torsionally softer blade configuration with $\omega_{T 1}=2.5$. Changes in right-rear skid microphone noise levels corresponding to the different control harmonics and phases are shown in Fig. 8. At this flight condition, every harmonic is capable of noise reduction when properly phased. Reductions of almost $9 \mathrm{~dB}$ are possible with $2 / r e v$ input and $5 \mathrm{~dB}$ with $3 / r e v$ input. The magnitude of these reductions is similar to those that could be obtained with the stiffer $\omega_{T 1}=3.17$ blade.

The effects of flap deflection on vibration levels for the torsionally softer blade is shown in Fig. 9 It is apparent from this figure that any of the four tested control harmonics is capable of reducing vibrations from the baseline if the control input is properly phased. A reduction of up to $80 \%$ can be achieved with $2 /$ rev or $4 /$ rev inputs. 

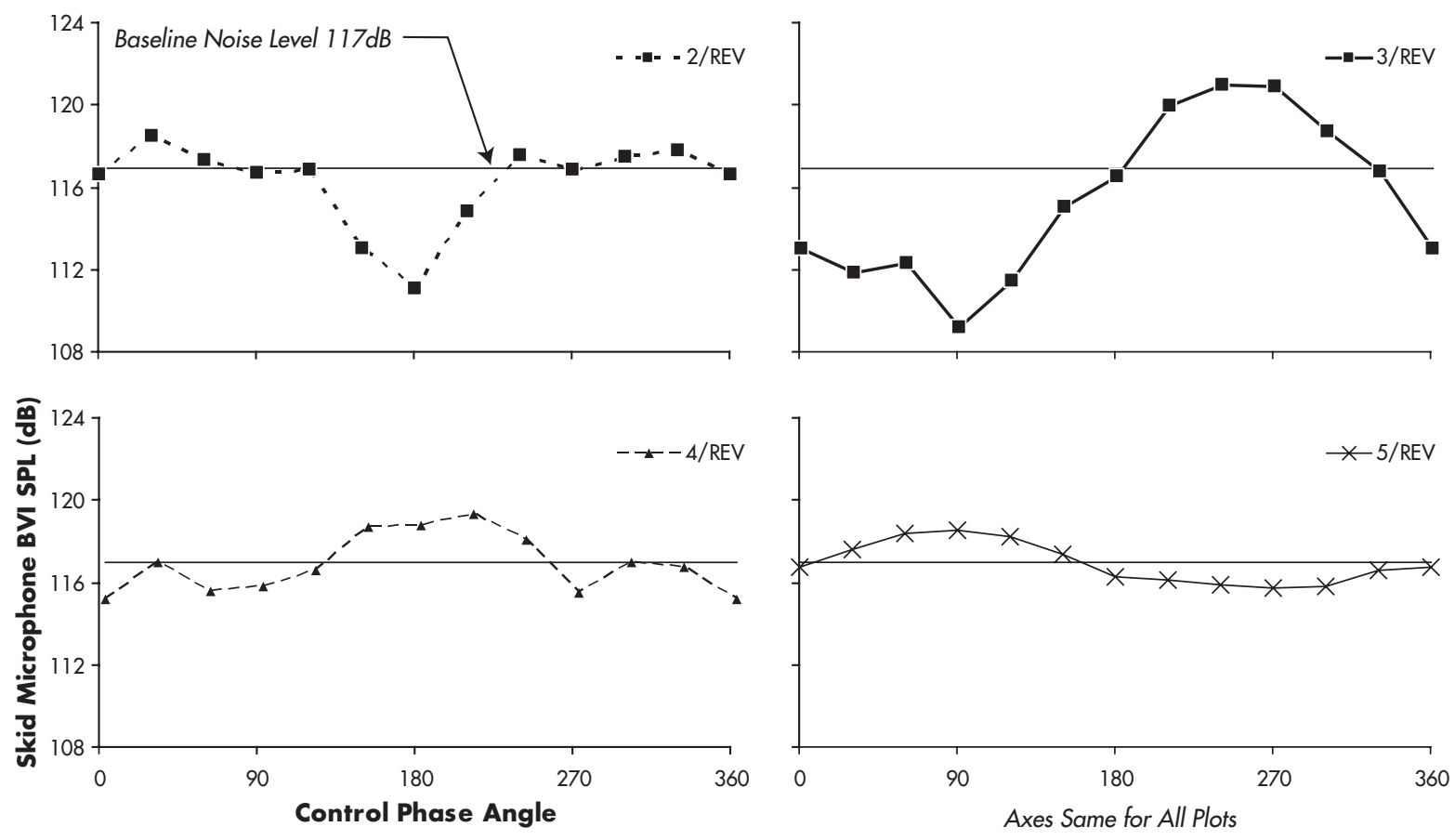

Figure 5. Effects of $4^{\circ}$ phase sweeps at 2,3,4 and $5 / \mathrm{rev}$ on BVI SPL at the right-rear skid microphone as compared to baseline noise level. MBB BO-105 rotor, single flap, $\mu=0.15,6^{\circ}$ descent, $\omega_{T 1}=3.17$.
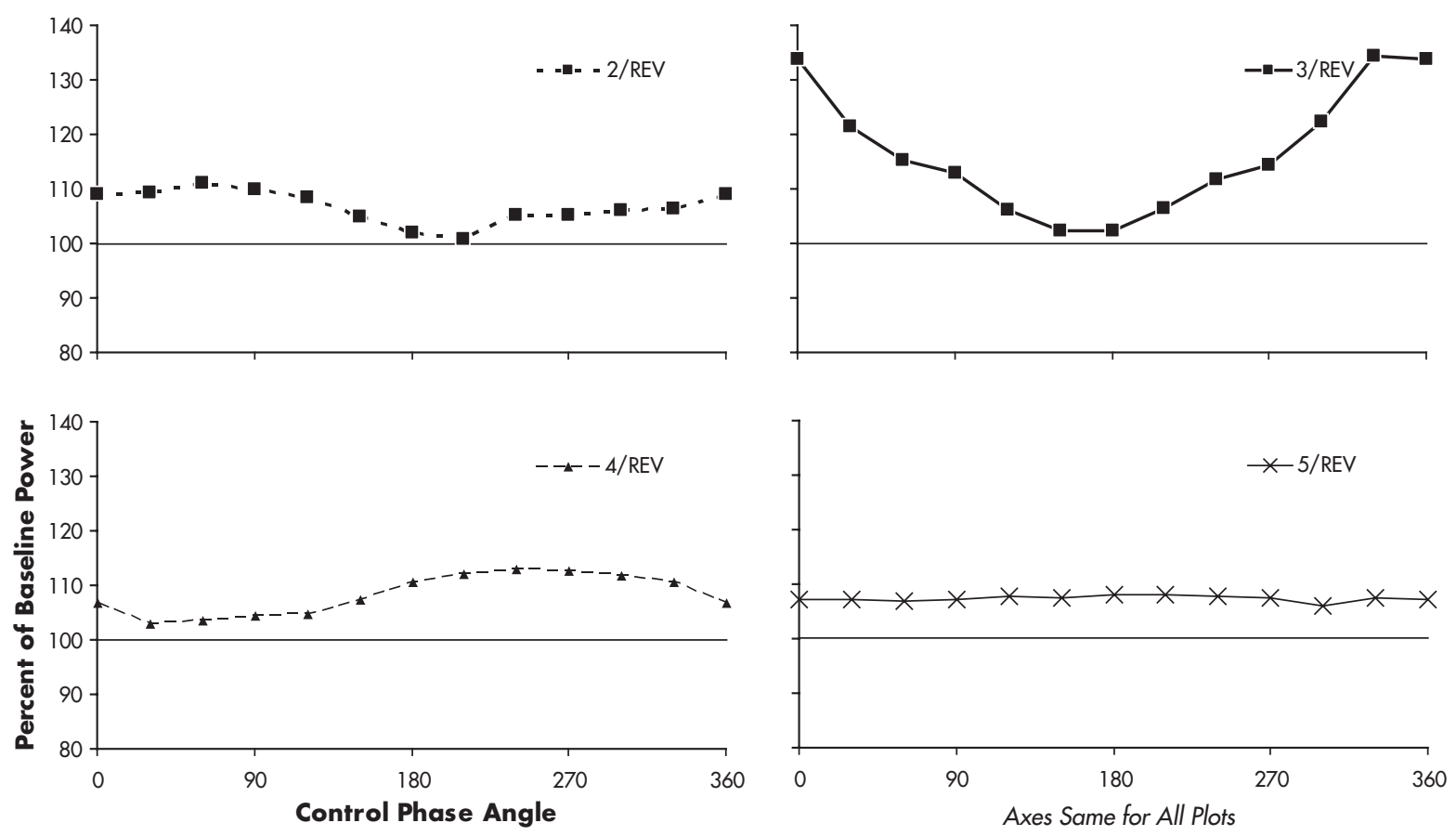

Figure 6. Effects of $4^{\circ}$ phase sweeps at 2,3,4 and 5/rev on rotor power $C_{P}$ as compared to baseline power. MBB BO-105 rotor, single flap, $\mu=0.15,6^{\circ}$ descent, $\omega_{T 1}=3.17$. 

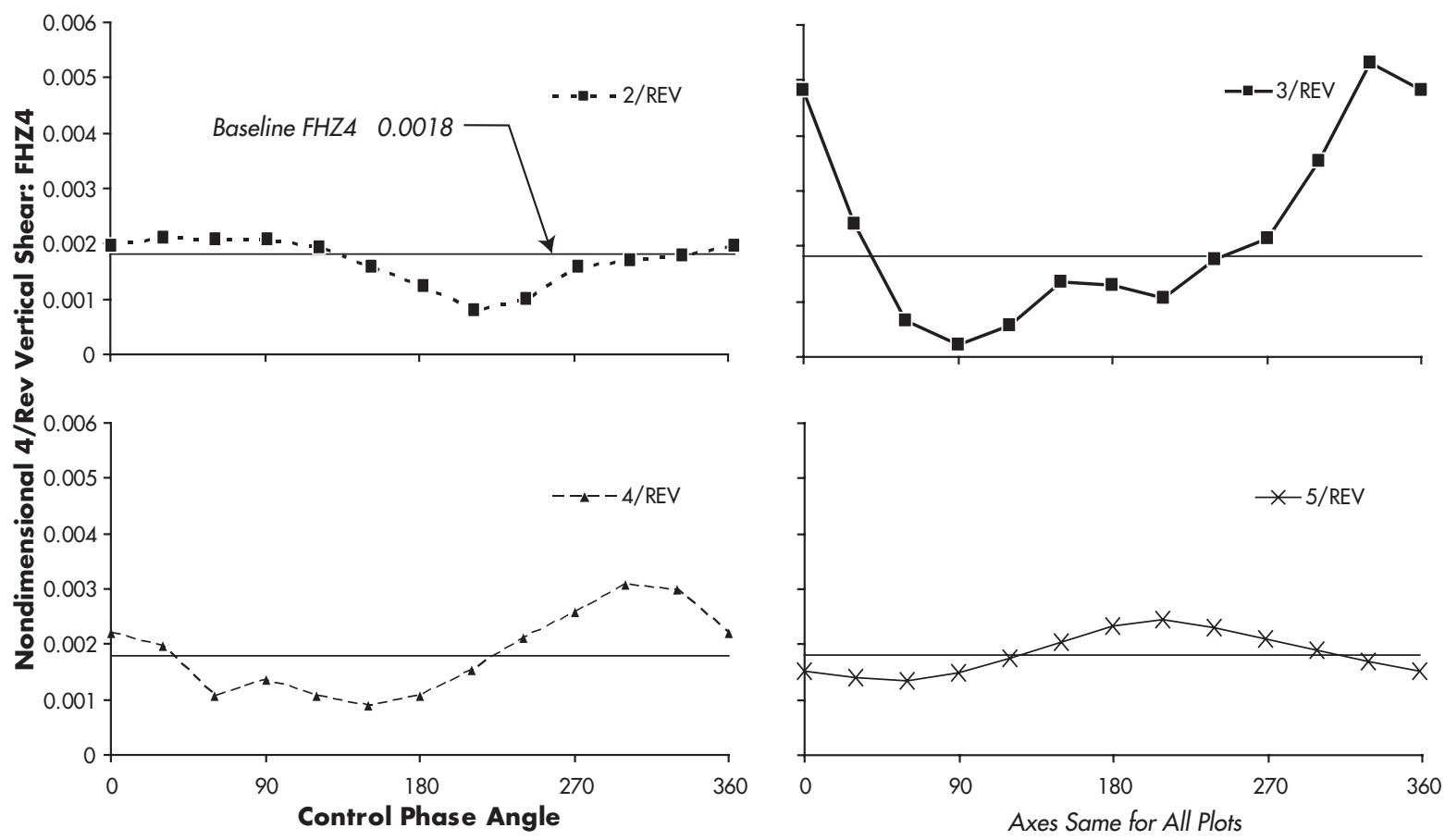

Figure 7. Effects of $4^{\circ}$ phase sweeps at 2,3,4 and $5 / \mathrm{rev}$ on vertical vibratory hub shear $F_{H Z 4}$ as compared to baseline. MBB BO-105 rotor, single flap, $\mu=0.15,6^{\circ}$ descent, $\omega_{T 1}=3.17$.
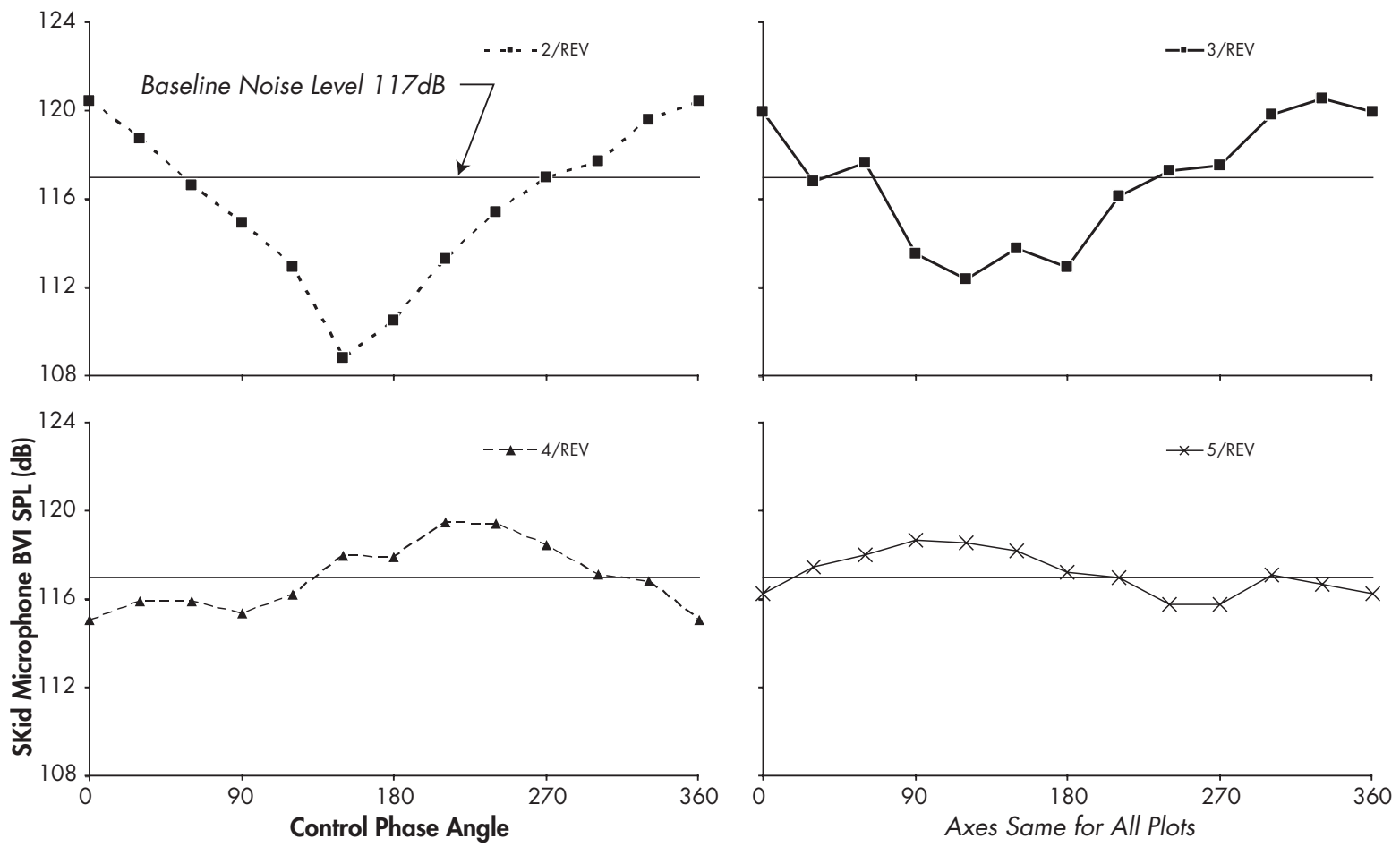

Figure 8. Effects of $4^{\circ}$ phase sweeps at 2,3,4 and 5/rev on BVI SPL at the right-rear skid microphone as compared to baseline noise level. Torsionally soft rotor, single flap, $\mu=0.15,6^{\circ}$ descent, $\omega_{T 1}=2.5$. 

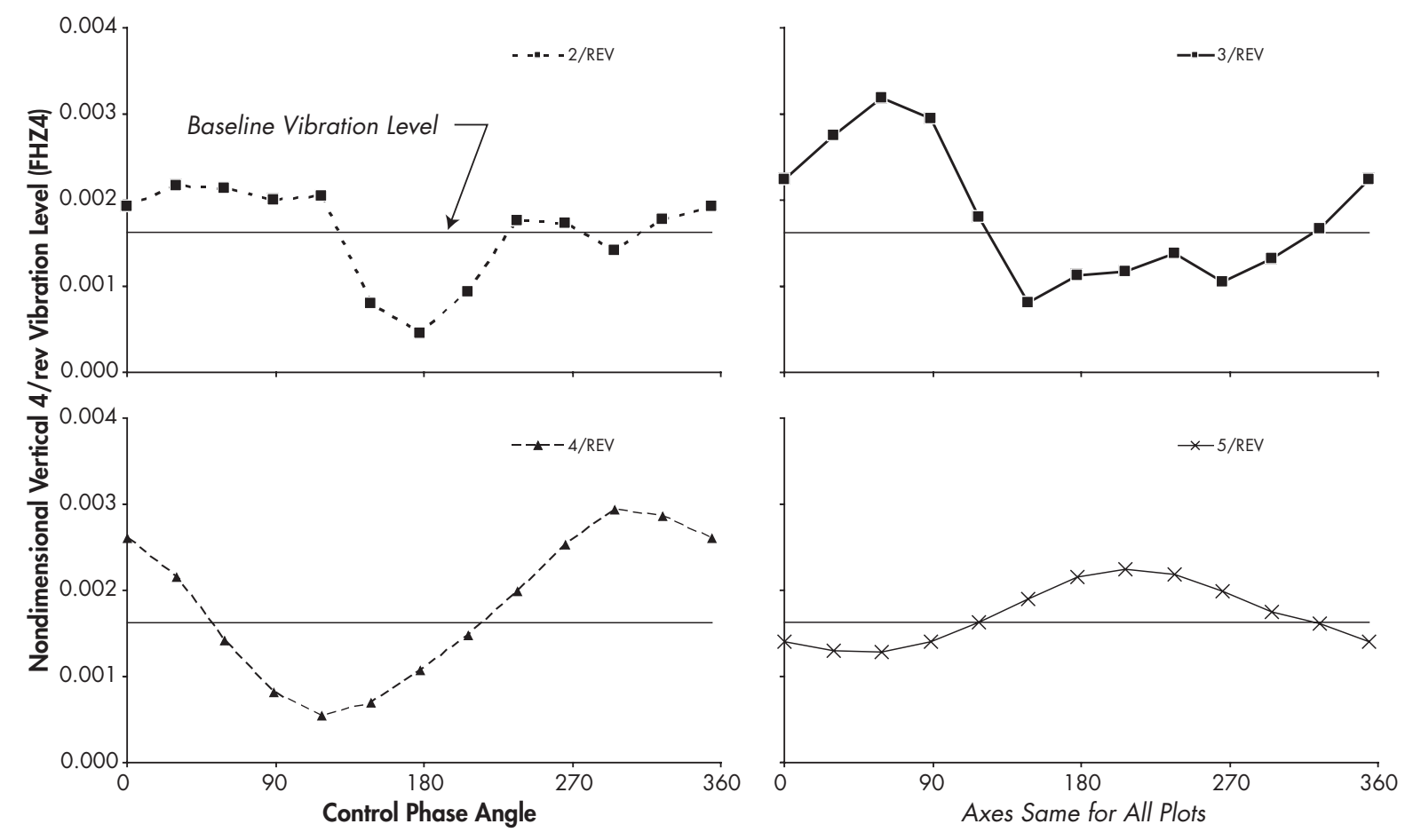

Figure 9. Effects of $4^{\circ}$ phase sweeps at $2,3,4$ and $5 / \mathrm{rev}$ on vertical vibratory hub shear $F_{H Z 4}$ as compared to baseline. Torsionally soft rotor, single flap, $\mu=0.15,6^{\circ}$ descent, $\omega_{T 1}=2.5$.

Finally, Fig. 10 shows the resulting changes in required rotor power for the torsionally soft blade with $\omega_{T 1}=2.5$. It is interesting to note that, unlike the MBB BO- 105 blade with $\omega_{T 1}=3.17$, two control phases of $2 / \mathrm{rev}$ and $3 / \mathrm{rev}$ are capable of reducing rotor power required by small amounts, up to $3.4 \%$ with $3 / \mathrm{rev}$ at $240^{\circ}$. Minor noise and vibration level reductions were also recorded with this control input.

\section{B. MBB BO-105 blade response with root-actuated IBC}

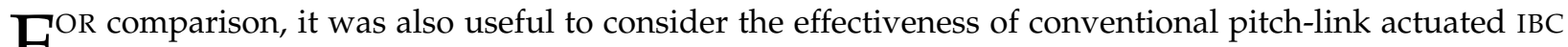
$\mathrm{F}$ at reducing BVI noise, vibration, and rotor power for the MBB BO-105 rotor configuration as given in Table 1 but without flaps. This open-loop control simulation is qualitatively similar to the wind-tunnel experiment described in Refs. 21 and 40; however, the results are not directly comparable due to different flight conditions, blade properties, and differing noise and vibration metrics. For the following open-loop tests, $1^{\circ}$ was chosen as the open-loop input pitch amplitude. The input phase is varied from $0^{\circ}$ to $360^{\circ}$ in $30^{\circ}$ increments for input frequencies of 2,3,4 and $5 /$ rev.

The resulting noise levels are shown in Fig. 11, and vibration levels are shown in Fig. 12. In Fig. 11, it is apparent that each of the input frequencies $2,3,4$ and $5 /$ rev is capable of reducing noise. The maximum noise reduction obtained was using a $3 /$ rev input at $180^{\circ}$, which produced a noise reduction of about $6 \mathrm{~dB}$. All input frequencies were capable of reducing vibration levels if phased properly (Fig. 12). However, the degree of vibration reduction obtained with IBC in this descent flight condition was not as good as the MBB BO-105 configuration with an actively controlled flap.

Figure 13 shows the resulting changes in required rotor power for the MBB BO-105 type blade with $\omega_{T 1}=3.17$ and IBC control. Note that both $2 / \mathrm{rev}$ and $4 / \mathrm{rev}$ control inputs are capable of reducing the power required to operate the rotor by as much as $3 \%$. However, conventional IBC control actuation has significantly higher power requirements than ACF actuation, ${ }^{41}$ and such an input could consume an additional $1-2 \%$ of the available engine power. Overall, the ACF has equal or better noise and vibration reduction 

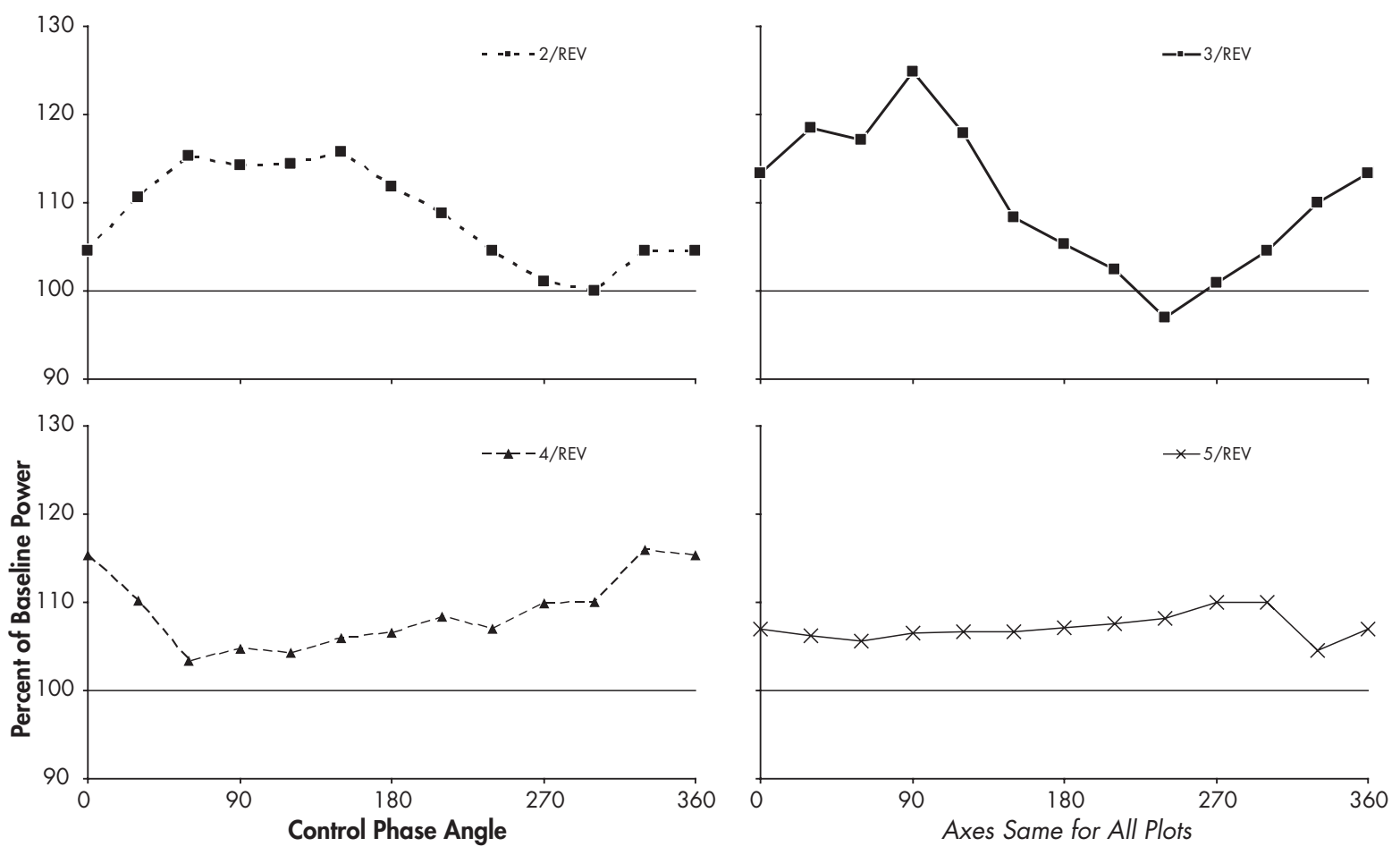

Figure 10. Effects of $4^{\circ}$ phase sweeps at $2,3,4$ and $5 / \mathrm{rev}$ on rotor power $C_{P}$ as compared to baseline power. Torsionally soft rotor, single flap, $\mu=0.15,6^{\circ}$ descent, $\omega_{T 1}=2.5$.
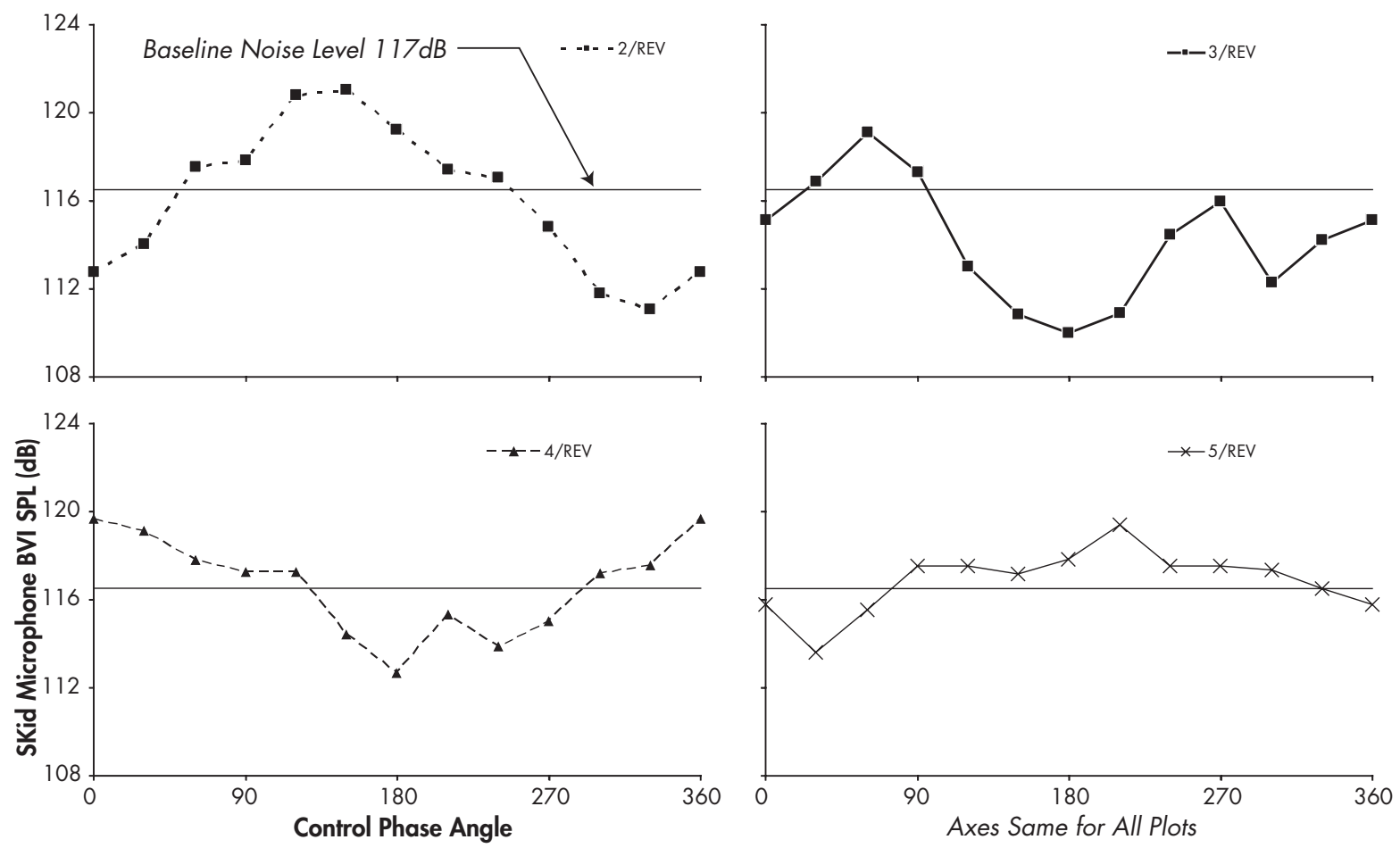

Figure 11. Effects of $1^{\circ}$ phase sweeps at 2,3,4 and $5 /$ rev on BVI SPL at the right-rear skid microphone as compared to baseline noise level. MBB BO-105 rotor, pitch-link IBC, $\mu=0.15,6^{\circ}$ descent, $\omega_{T 1}=3.17$. 

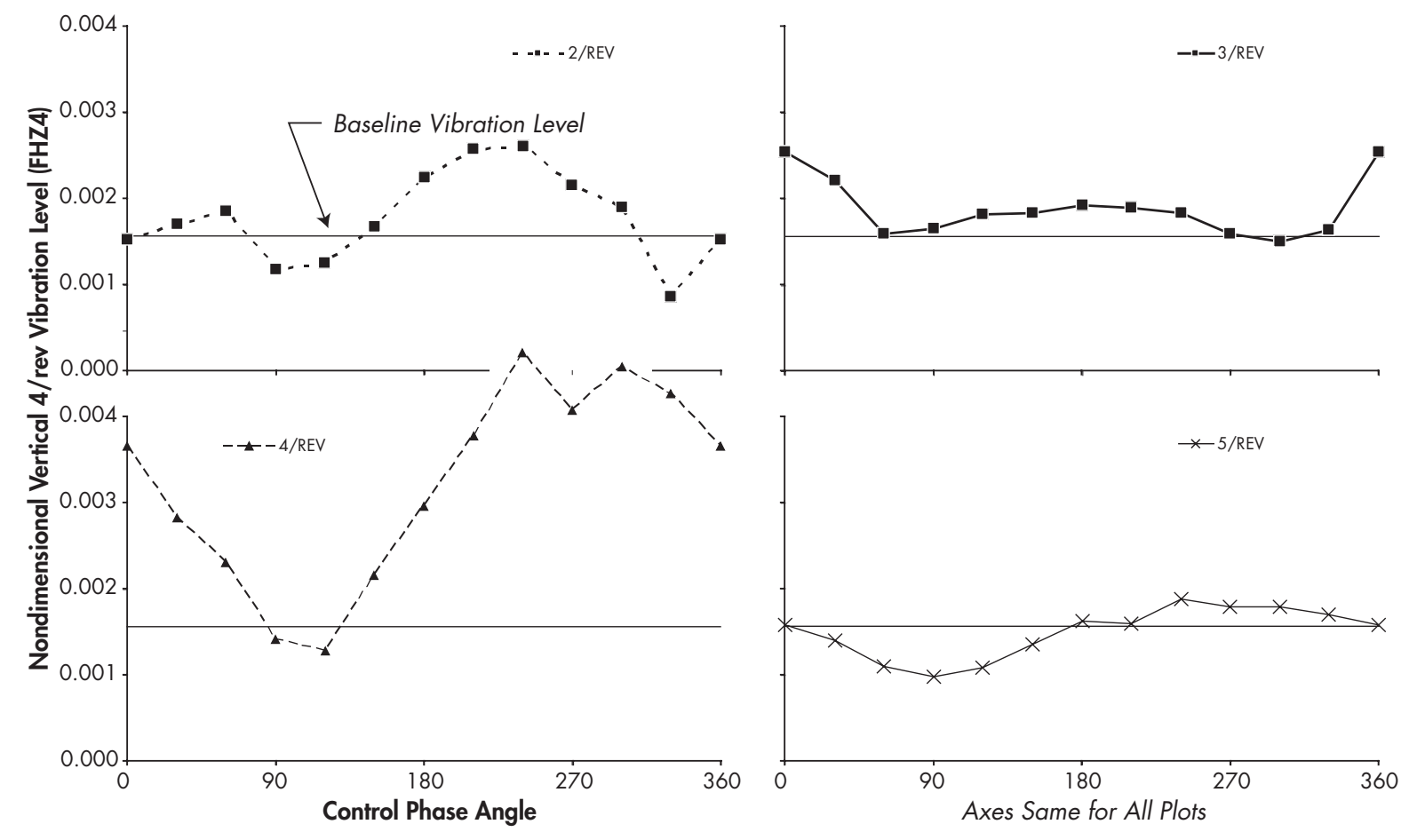

Figure 12. Effects of $1^{\circ}$ phase sweeps at $2,3,4$ and $5 /$ rev on vertical vibratory hub shear $F_{H Z 4}$ as compared to baseline power. MBB BO-105 rotor, pitch-link IBC, $\mu=0.15,6^{\circ}$ descent, $\omega_{T 1}=3.17$.

capability when compared to IBC for this configuration and flight condition.

\section{Noise Reduction and Directivity in the Far-Field}

NOTHER interesting aspect of the problem is associated with the examination of the magnitude and A directivity of BVI noise before and after closed-loop control with a dual ACF configuration on a MBB BO-105 rotor. The sound field around the rotor is determined by considering sound pressure levels (SPLs) on three carpet planes beneath the rotor, as depicted in Fig. 14. Previous studies ${ }^{2}$ have only considered noise on the primary carpet plane. The closed-loop control technique used for this study is the adaptive higher-harmonic control (HHC) algorithm described extensively in Refs. 28 and 42.

The baseline noise levels on the primary carpet plane are shown in Fig. 15 a. Note that the designations a-f distinguishing between the subfigures is provided in the upper left hand corner. The noise levels for the dual servo flap configuration with $4^{\circ}$ saturation limits is shown in Fig. 15d. On the primary carpet plane, a reduction of $5-7 \mathrm{~dB}$ is achieved on the advancing side, with no retreating side noise penalty. Noise levels on the second carpet plane are shown in Fig. $15 \mathrm{p}$ for the baseline case and in Fig. 15p for the controlled case. On the second plane, peak advancing side noise levels are reduced by $3-5 \mathrm{~dB}$, and retreating side noise levels by $1-2 \mathrm{~dB}$. Noise levels on the third carpet plane are shown in Fig. 15 f for the baseline case and in Fig. 15f for the controlled case. On the third carpet plane, advancing side noise levels are reduced by 3-5dB, and retreating side noise levels by $1-2 \mathrm{~dB}$. Further, the sizes of the peak advancing and retreating noise lobes are significantly reduced. It is interesting to note that although no noise reduction on the retreating side was apparent on the primary carpet plane (which is still in the acoustic near-field), a significant reduction is apparent in the far-field. These results demonstrate that the ACF has great potential as a noise-reduction device, as it can substantially lower noise levels far beneath the rotor. 

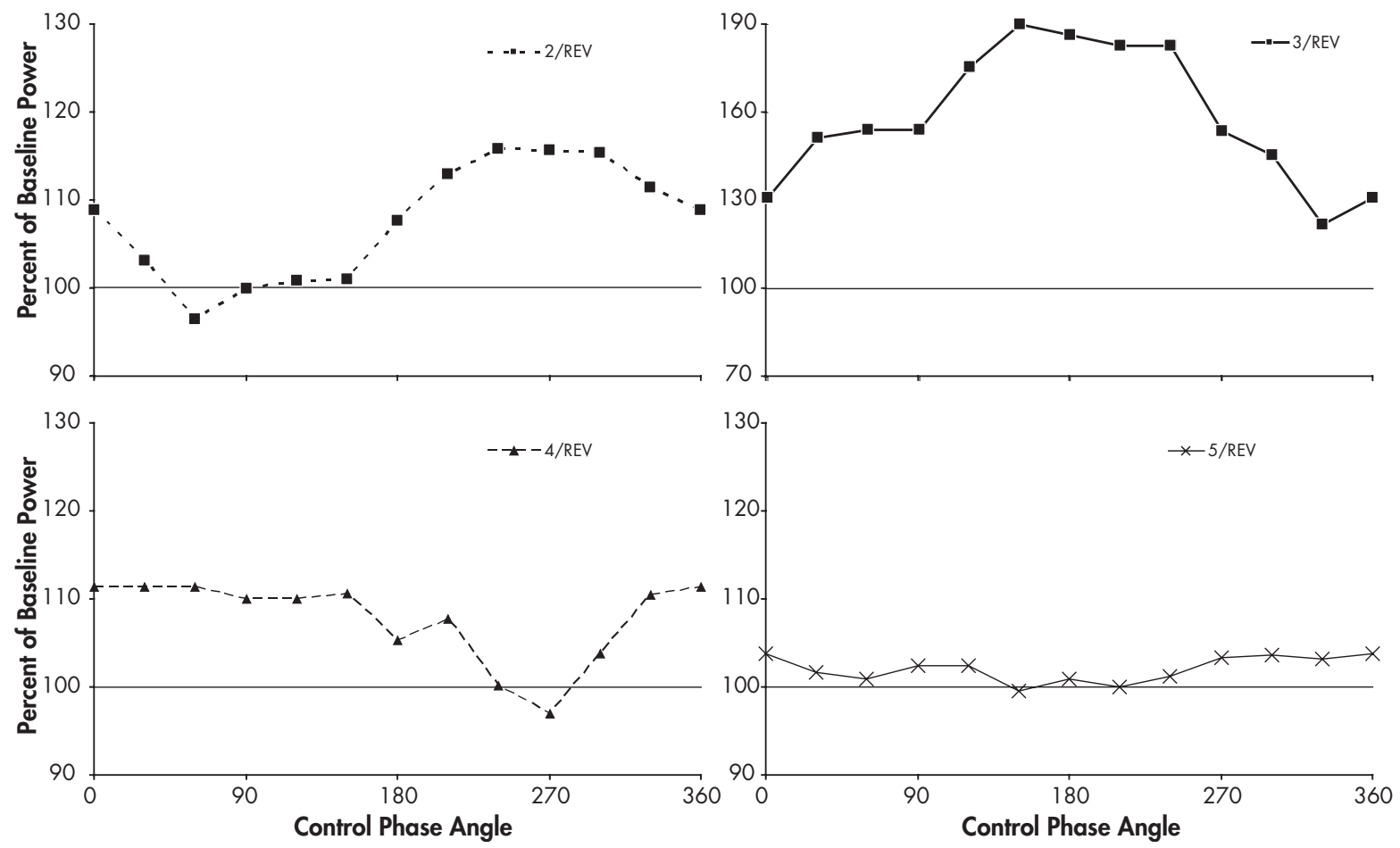

Figure 13. Effects of $1^{\circ}$ phase sweeps at 2,3,4 and 5/rev on rotor power $C_{P}$ as compared to baseline power. MBB BO-105 rotor, pitch-link IBC, $\mu=0.15,6^{\circ}$ descent, $\omega_{T 1}=3.17$.

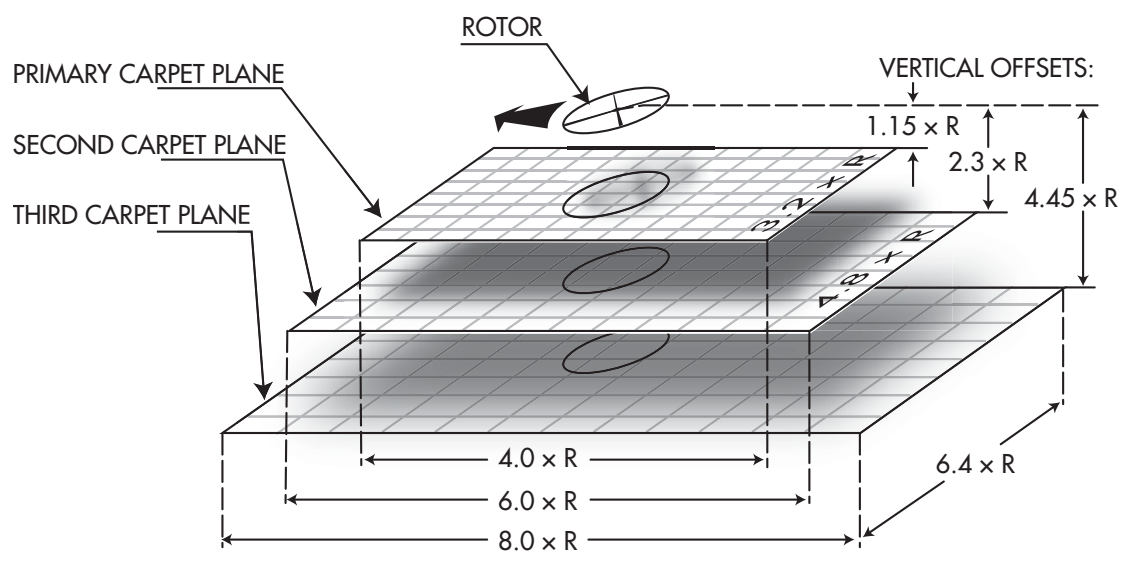

Figure 14. Three carpet planes of increasing size and distance from the main rotor 

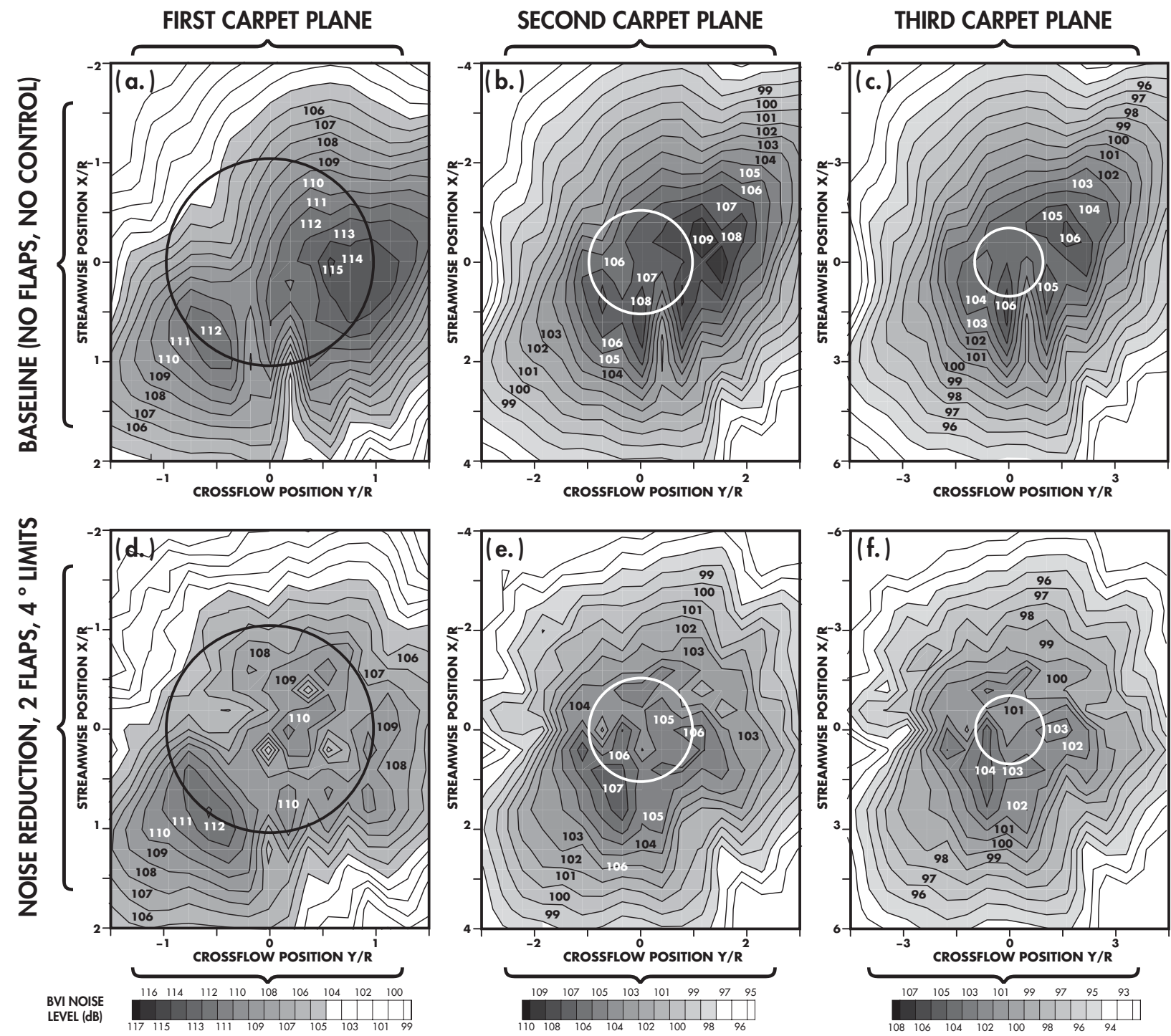

Figure 15. BVI sound pressure levels on the three carpet planes for the baseline case and a dual-flap case, with $4^{\circ}$ saturation limits 


\section{Vibration, Noise and Simultaneous Reduction on a MD-900 Rotor}

CTIVE closed-loop control studies have previously demonstrated noise, vibration, and simultaneous A noise and vibration reduction on a four-bladed MBB BO-105 bearingless rotor. ${ }^{2,27,28}$ In this section, the five-bladed MD-900 SMART bearingless rotor configuration, as described in Table 1 is considered.

\section{Vibration Reduction}

Initially, the active flap is used for vibration reduction alone. The adaptive HHC control algorithm ${ }^{42}$ attempts to reduce an objective function consisting of $5 / \mathrm{rev}$ vibratory hub loads since the main rotor is 5 bladed and $5 / \mathrm{rev}$ vibratory components dominate. The flap deflection frequency components of interest for active control are $2 / \mathrm{rev}, 3 / \mathrm{rev}, 4 / \mathrm{rev}, 5 / \mathrm{rev}, 6 / \mathrm{rev}$. Moreover, flap deflection saturation limits are enforced, constraining motion to $\pm 4^{\circ}$, thereby complying with design specifications of the active flap.

Figure 16 a shows $5 /$ rev vibratory hub loads, for the baseline (uncontrolled) case and after vibration control. The active flap produces vibration reduction of nearly $50 \%$ in simulated descent flight in the presence of heavy BVI. This demonstrates the effectiveness of the ACF on the five-bladed bearingless rotor. The time history of flap deflection for this control case is shown in Figure 18 a.

The effects of vibration reduction on noise were also considered. Noise carpet plots at $1.15 R$ below the hub plane are shown in Figs. 17a and 17k for the baseline and vibration control cases, respectively. Similar results were achieved with the MBB BO-105 configuration (Figs. $17 \mathrm{p}, \mathrm{d}$ and e) ${ }^{2}$ the peak advancing side noise is increased by about $1 \mathrm{~dB}$ during vibration reduction.

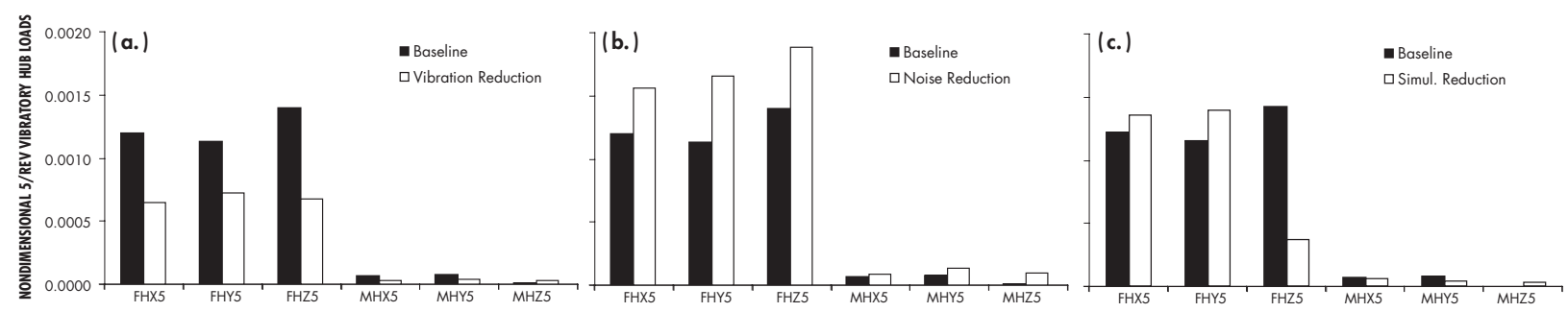

Figure 16. 5/rev vibratory loads, the baseline and with vibration reduction

\section{Noise Reduction}

The same configuration was considered with a modified control objective function aimed at reducing BVI noise levels ${ }^{2}$ at the SKID-REAR microphone location, with flap input frequency components of 2-6/rev. The peak advancing side noise at the carpet plane is reduced by $3 \mathrm{~dB}$, as can been from Fig. 17f. It should be noted that retreating side noise does not increase for this case, unlike the single flap MBB BO- 105 results shown in Fig. 17g. The BVI noise is seen to be reduced uniformly over the entire carpet plane. This demonstrates the effectiveness of ACF for noise reduction on the bearingless configuration. The flap deflection time history is shown in Fig. 18 p.

The vibratory loads during noise reduction are presented in Fig. 16p. It can been seen that the noise reduction is associated with a significant vibration penalty. All vibratory components are increased by about $20-30 \%$. This is similar to the noise control case for the four-bladed hingeless MBB BO-105 rotor. ${ }^{2}$

\section{Simultaneous Reduction}

Simultaneous vibration and noise reduction using the active flap is also considered, with flap frequency components of 2-6/rev. The vibratory loads and noise carpet plots after simultaneous control are shown in Figs. 16. and 17, respectively. Although the longitudinal and lateral hub shears are increased slightly after control, the vertical vibration component is significantly reduced by $74 \%$ and the resulting vibration objective is reduced by about $8 \%$. Meanwhile, BVI noise is reduced by $1-2 \mathrm{~dB}$. Overall, the combined vibration and noise objective is reduced by about $30 \%$, representing a compromise solution. Comparing these with the simultaneous noise and vibration reduction observed on a hingeless rotor similar to the MBB 

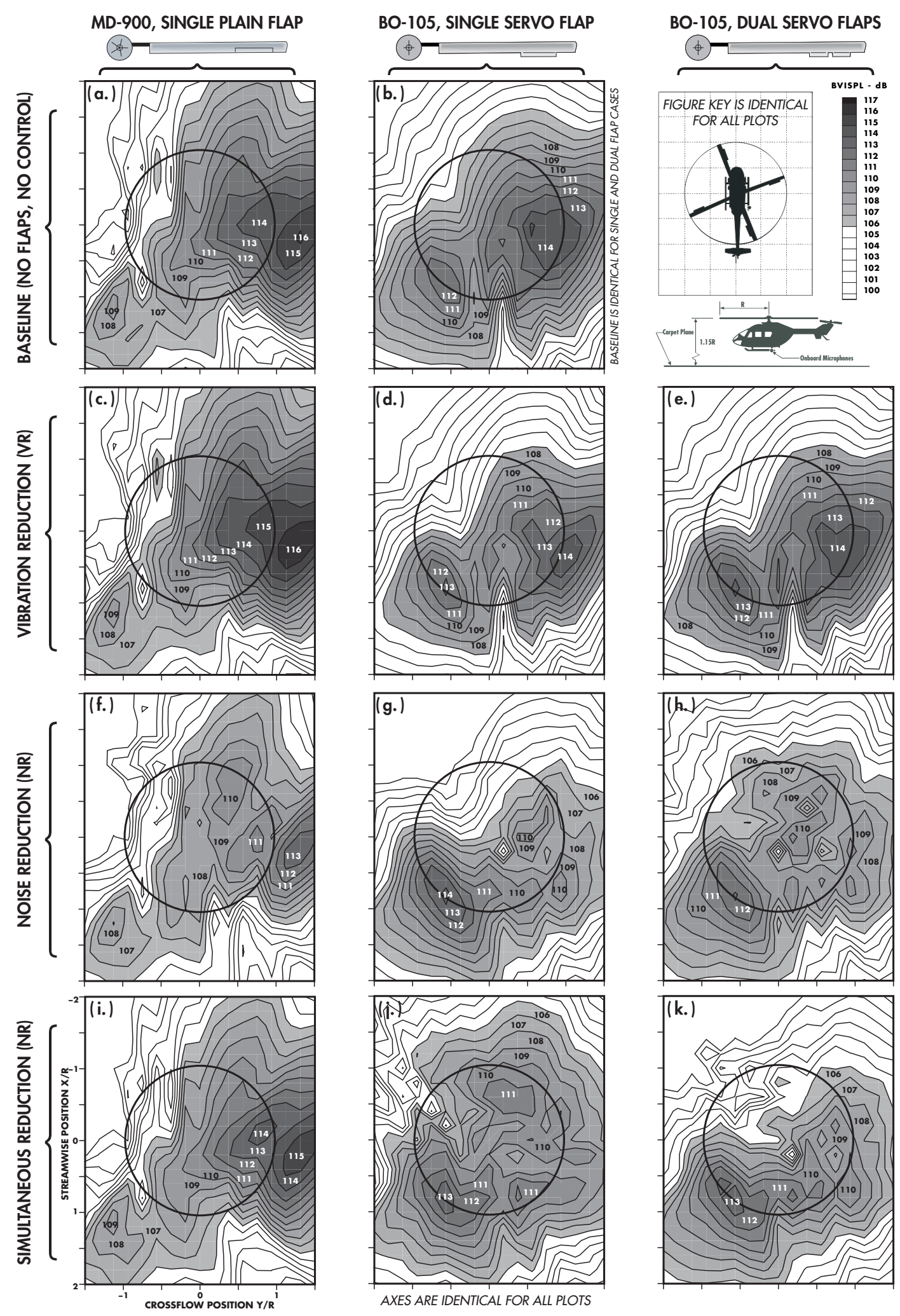

Figure 17. Noise carpet plots, MD-900 and BO-105 Configurations 

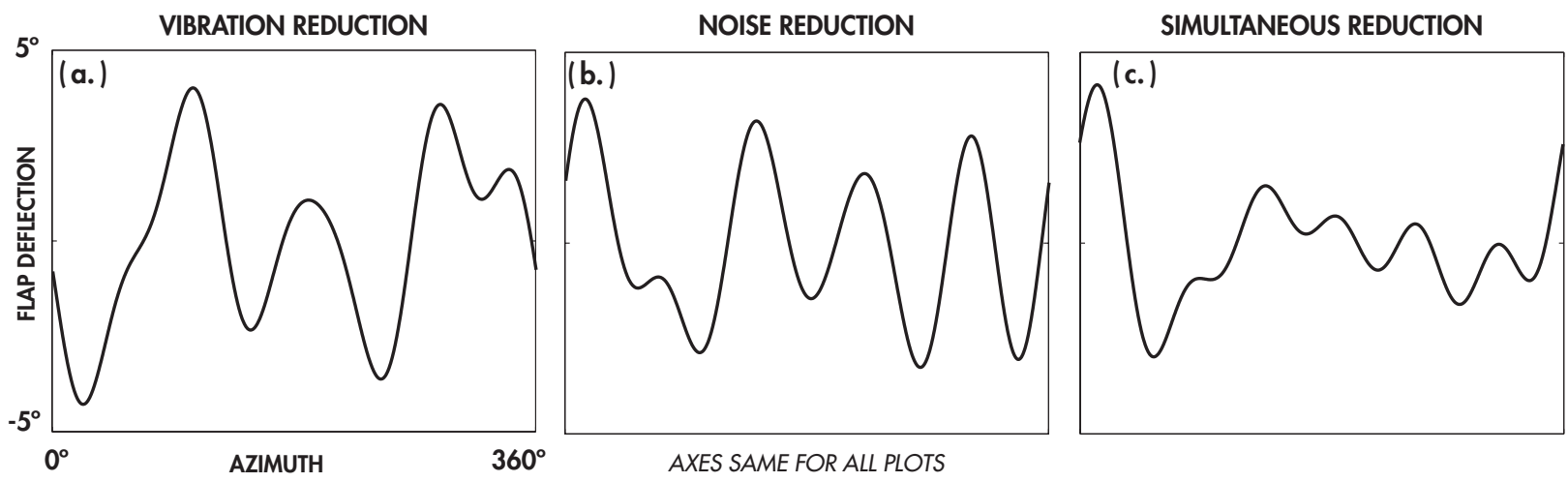

Figure 18. Flap deflection during vibration reduction

BO-105, 2,9 it appears that the noise reductions obtained for the bearingless rotor are smaller. However, this may also be due to the use of the plain flap, for the bearingless rotor case, which is known to be less effective than the servo flap based ACF system used for the other cases studied.

\section{Concluding Remarks}

THE actively controlled flap holds considerable promise as a means of reducing noise, vibration, and perhaps rotor power. Using numerical simulations, the effectiveness of this device has been demonstrated for several rotor and flap configurations, as summarized in Table 3

Table 3. Summary of key results from two rotor configurations, with $4^{\circ}$ saturation limits.

\begin{tabular}{|c|c|c|c|c|c|c|c|c|c|}
\hline Rotor & \multicolumn{6}{|c|}{ BO-105 (Ref. 2) } & \multicolumn{3}{|c|}{ MD-900 } \\
\hline Type & \multicolumn{6}{|c|}{ Hingeless } & \multirow{3}{*}{\multicolumn{3}{|c|}{$\begin{array}{l}\text { Bearingless } \\
5 \\
\text { single plain }\end{array}$}} \\
\hline$N_{b}$ & & & & 4 & & & & & \\
\hline Flap No. \& Type & \multicolumn{3}{|c|}{ single servo } & \multicolumn{3}{|c|}{ dual servo } & & & \\
\hline Controller Objective & VR & NR & SR & VR & NR & SR & VR & NR & SR \\
\hline$\Delta$ Vibration $^{+}$ & $-46 \%$ & $+2 \%$ & $-57 \%$ & $-86 \%$ & $+130 \%$ & $-44 \%$ & $-60 \%$ & $+150 \%$ & $-8 \%$ \\
\hline$\Delta$ BVI Noise ${ }^{\ddagger}$ & $-1 \mathrm{~dB}$ & $-6 \mathrm{~dB}$ & $-3 d B$ & $-1 d B$ & $-5 \mathrm{~dB}$ & $-4 \mathrm{~dB}$ & $+1 \mathrm{~dB}$ & $-4 \mathrm{~dB}$ & $-2 d B$ \\
\hline
\end{tabular}

The results presented in this paper provide new insights on the effectiveness of flaps for noise and vibration reduction. The principal observations and conclusions are summarized below:

1. The ACF is effective for advancing-side BVI noise reduction, identified to be most annoying to observers on the ground. Open-loop control studies indicate that $2,3,4$ or $5 / \mathrm{rev}$ inputs can be used to reduce noise, although $2 / \mathrm{rev}$ and $3 / \mathrm{rev}$ are most effective.

2. The open-loop inputs which are most effective at noise reduction are not the same as those which are most effective at vibration reduction.

3. With rotor blades sufficiently soft in torsion, it appears that a rotor power reduction of up to $3 \%$ is possible using the ACF and a control input of $3 / \mathrm{rev}$ at $240^{\circ}$. Noise and vibration levels were also observed to drop at this input condition. 
4. The ACF and conventional IBC are equally effective at noise reduction in descending BVI flight conditions. The ACF is more effective at reducing vibration, however. Although it appears that conventional IBC may be slightly more effective at rotor power reduction, these gains could be offset by higher control system power requirements.

5. The ACF was observed to be effective at reducing BVI noise in both the near-field and far-field when using feedback control based on a skid-mounted microphone. Both advancing and retreating side noise levels were reduced by up to $5 d B$ in the far-field.

6. The ACF is effective at reducing noise and vibration on a five-bladed bearingless rotor. Noise reductions of $4 \mathrm{~dB}$ or vibration reductions of $50 \%$ could be achieved. A compromise simultaneous reduction was also possible.

\section{Acknowledgments}

This research was supported in part by a Rackham Predoctoral Fellowship from the University of Michigan for D. Patt, and by ARO grant 02-1-0202 with Dr. G. Anderson as grant monitor. Partial support by the FXв Center for Rotary and Fixed Wing Air Vehicle Design is also gratefully acknowledged. The authors would also like to thank Dr. Yung H. Yu of the AFDD for providing HART test data.

\section{References}

${ }^{1}$ Yu, Y. H., "Rotor Blade-Vortex Interaction Noise," Progress in Aerospace Sciences, Vol. 36, No. 2, Feb. 2000, pp. $97-115$.

${ }^{2}$ Patt, D., Liu, L., and Friedmann, P. P., "Achieving Simultaneous Noise and Vibration Reduction Using Simulation," Proceedings of the 30th European Rotorcraft Forum, Marseille, France, September 2004.

${ }^{3}$ Splettstoesser, W., Kube, R., Wagner, W., Seelhorst, U., Boutier, A., Micheli, F., Mercker, E., and Pengel, K., “Key Results From a Higher Harmonic Control Aeroacoustic Rotor Test (HART)," Journal of the American Helicopter Society, Vol. 42, No. 1, Jan. 1997, pp. 5878.

${ }^{4} J a c k l i n$, S. A., Haber, A., de Simone, G., Norman, T., Kitaplioglu, C., and Shinoda, P., "Full-Scale Wind Tunnel Test of an Individual Blade Control System for a UH-60 Helicopter," Proceedings of the 51st Annual Forum of the American Helicopter Society, Montreal, Canada, June 2002.

${ }^{5}$ Roth, D., "Advanced Vibration Reduction by IBC Technology," Proceedings of the 30th European Rotorcraft Forum, Marseille, France, September 2004.

${ }^{6}$ Millott, T. A. and Friedmann, P. P., Vibration Reduction in Helicopter Rotors Using an Actively Controlled Partial Span Trailing Edge Flap Located on the Blade, NASA CR 4611, June 1994.

${ }^{7}$ Depailler, G. and Friedmann, P. P., "Reductions of Vibrations Due to Dynamic Stall in Helicopters Using an Actively Controlled Flap," Proceedings of the 43rd AIAA/ASME/ASCE/AHS/ACS Structures, Structural Dynamics and Materials Conference, Denver, CO, April 2002, AIAA Paper No. 2002-1431.

${ }^{8}$ Patt, D., Liu, L., and Friedmann, P. P., “Rotorcraft Vibration Reduction and Noise Prediction Using a Unified Aeroelastic Response Simulation," Proceedings of the 59th American Helicopter Society Annual Forum, Phoenix, AZ, May 2003.

${ }^{9}$ Liu, L., Patt, D., and Friedmann, P. P., "Simultaneous Vibration and Noise Reduction in Rotorcraft Using Aeroelastic Simulation," Proceedings of the 60th American Helicopter Society Annual Forum, Baltimore, MD, June 2004.

${ }^{10}$ Schmitz, F. H., Rotor Noise, Aeroacoustics of Flight Vehicles, Vol.1, Acoustical Society of America and American Institute of Physics, New York, 1995, pp. 65-149.

${ }^{11}$ Leishman, J. G., "Sound Directivity Generated By Helicopter Rotors Using Wave Tracing Concepts," Journal of Sound and Vibration, Vol. 221, No. 3, 1999, pp. 415-441.

${ }^{12}$ Schmitz, F. H. and Sim, B. W., "Radiation and Directionality Characteristics of Helicopter Blade-Vortex Interaction Noise," Journal of the American Helicopter Society, Vol. 48, No. 4, Oct. 2003, pp. 253-269.

${ }^{13}$ Brentner, K. S. and Farassat, F., "Modeling Aerodynamically Generated Sound of Helicopter Rotors," Progress in Aerospace Sciences, Vol. 39, No. 2-3, February-April 2003, pp. 83-120.

${ }^{14}$ Strawn, R., “Unstructured Adaptive Mesh Computations of Rotorcraft High-Speed Impulsive Noise," Journal of the American Helicopter Society, Vol. 32, No. 4, July 1995, pp. 754-760.

${ }^{15}$ Farassat, F. and Brentner, K. S., "Supersonic Quadrupole Noise Theory for High-Speed Helicopter Rotors," Journal of Sound and Vibration, Vol. 218, No. 3, 1998, pp. 481-500.

${ }^{16}$ Bres, G. A., Brentner, K., Perez, G., and Jones, H. E., “Helicopter Noise Prediction: The Current Status and Future Direction," Journal of Sound and Vibration, Vol. 170(1), 1994, pp. 79-96.

${ }^{17}$ Brooks, T. F. and Burley, C. L., "Blade Wake Interaction Noise for a Main Rotor," Journal of the American Helicopter Society, Vol. 49 , No. 1, Jan. 2004, pp. 11-27. 
${ }^{18}$ Burley, C. L. and Brooks, T. F., "Rotor Broadband Noise Prediction with Comparison to Model Data," Journal of the American Helicopter Society, Vol. 49, No. 1, Jan. 2004, pp. 28-42.

${ }^{19}$ Ffowcs Williams, J. E. and Hawkings, D. L., "Sound Generation by Turbulence and Surfaces in Arbitrary Motion," Philosophical Transactions of Royal Soc. London, Series A, Vol. 264, No. 1151, May 1969, pp. 321-342.

${ }^{20}$ van der Wall, B. G., Junker, C., Burley, C., Brooks, T., Yu, Y., Raffel, P., and Wagner, W., "The HART II test in the LLF of the DNW - a Major Step towards Rotor Wake Understanding," Proceedings of the 28th European Rotorcraft Forum, Bristol, England, Sept. 2002.

${ }^{21}$ Swanson, S. M., Jacklin, S. A., Blaas, ., Niesl, G., and Kube, R., "Reduction of Helicopter BVI Noise, Vibration, and Power Consumption through Individual Blade Control," Proceedings of the 51st Annual Forum of the American Helicopter Society, Fort Worth, TX, May 1995, pp. 662-680.

${ }^{22}$ Bebesel, M., Roth, D., and Pongratz, K., "Reduction of BVI Noise on the Ground - In Flight Evaluation of Closed-Loop Controller," Proceedings of the 28th European Rotorcraft Forum, Bristol, England, Sept. 2002, pp. 19.1-19.9.

${ }^{23}$ Booth, E. R. and Wilbur, M. L., "Acoustic Aspects of Active-Twist Rotor Control," Proceedings of the American Helicopter Society 58th Annual Forum, Montreal, Canada, June 2002.

${ }^{24}$ Malovrh, B. and Gandhi, F., "Localized Individual Pitch Control for Reduction of Helicopter Blade-Vortex Interaction Noise," Proceedings of the 28th European Rotorcraft Forum, Bristol, England, Sept. 2002, pp. 6.1-6.10.

${ }^{25} \mathrm{~F}$. Gandhi and L. Tauszig, "A Method to Evaluate the Contributions of Individual Interactions to Helicopter Blade-Vortex Interaction Noise," Journal of the American Helicopter Society, Vol. 48, No. 4, Oct. 2003, pp. 287-299.

${ }^{26}$ Tauszig, L., Analysis of Helicopter Blade-Vortex Interaction Noise With Application to Adaptive-Passive and Active Alleviation Methods, Ph.D. thesis, The Pennsylvania State University, 2002.

${ }^{27}$ Patt, D., Liu, L., and Friedmann, P. P., "Rotorcraft Vibration Reduction and Noise Prediction Using a Unified Aeroelastic Response Simulation," Journal of the American Helicopter Society, January 2005, pp. 95-106.

${ }^{28}$ Patt, D., Simultaneous Noise and Vibration Reduction in Rotorcraft Using Actively Controlled Flaps and Including Performance Considerations, Ph.D. thesis, University of Michigan, Ann Arbor, 2004.

${ }^{29}$ Lowson, M. V., “Focusing of Helicopter BVI Noise," Journal of Sound and Vibration, Vol. 190, No. 3, 1996, pp. 477-494.

${ }^{30}$ Martin, R. M., Splettstoesser, W. R., Elliot, J. W., and Schultz, K. J., Advancing Side Directivity and Retreating Side Interactions of Model Rotor Blade-Vortex Interaction Noise, NASA TP 2784, 1988.

${ }^{31}$ Sim, B. W., Schmitz, F. H., and Aoyama, T., “Near/Far-Field Radiation Characteristics of Advancing Side Helicopter BladeVortex Interaction (BVI) Noise," Proceedings of the American Helicopter Society 56th Annual Forum, Virginia Beach, VA, May 2000.

${ }^{32}$ Straub, F. K., Kennedy, D. K., Stemple, A. D., Anand, V., and Birchette, T. S., "Development and whirl tower test of the SMART active flap rotor," Proceedings of SPIE: Smart Structures and Materials 2004, Vol. 5388, July 2004, pp. 202-212.

${ }^{33}$ Yuan, K. A. and Friedmann, P. P., Aeroelastic and Structural Optimization of Composite Helicopter Rotor Blades with Swept Tips, NASA CR 4665, June 1995.

${ }^{34}$ USAAMCOM/AFDD TR 02-A-006, RCAS User's Manual, Version 2.0, June 2002.

${ }^{35}$ Brenter, K., A Computer Program Incorporating Realistic Blade Motions and Advanced Acoustic Formulation, NASA Technical Memorandum, Vol. 877211986.

${ }^{36}$ Johnson, W., "Rotorcraft Dynamics Models for a Comprehensive Analysis," Proceedings of the 54th Annual Forum of the American Helicopter Society, Washington, D.C., May 1998.

${ }^{37}$ Straub, F. K. and Charles, B. D., "Aeroelastic Analysis of Rotors with Trailing Edge Flaps Using Comprehensive Codes," Journal of the American Helicopter Society, Vol. 46, No. 3, July 2001, pp. 192-199.

${ }^{38}$ Cheng, R. P., Theodore, C., and Celi, R., "Effects of Higher Harmonic Control on Rotor Performance," Journal of the American Helicopter Society, Vol. 48, No. 1, January 2003, pp. 18-27.

${ }^{39}$ Cheng, R. P. and Celi, R., "Optimum 2/Rev Inputs for Improved Rotor Performance," Proceedings of the American Helicopter Society Northeast Region Active Controls Technology Conference, Fairfield County,Connecticut, October 2000.

${ }^{40}$ Jacklin, S. A., "Second Test of a Helicopter Individual Blade Control System in the NASA Ames 40 by 80 Foot Wind Tunnel," Proceedings of the 2nd International American Helicopter Society Aeromechanics Specialists Conference, Bridgeport, CT, Oct. 1995.

${ }^{41}$ Friedmann, P. P. and Millott, T. A., "Vibration Reduction in Rotorcraft Using Active Control: A Comparison of Various Approaches," Journal of Guidance, Control, and Dynamics, Vol. 18, No. 4, July-August 1995, pp. 664-673.

${ }^{42}$ Patt, D., Liu, L., Chandrasekar, J., Bernstein, D. S., and Friedmann, P. P., “The Higher Harmonic Algorithm for Helicopter Vibration Reduction Revisited," Proceedings of the 45th AIAA/ASME/ASCE/AHS/ACS Structures, Structural Dynamics and Materials Conference, Palm Springs, CA, April 2004, AIAA Paper No. 2004-1948. To appear in the Journal of Guidance, Control and Dynamics, 2005. 